\title{
Lebensraum-Hotspots für saproxylische Arten mittels LFI-Daten erkennen
}

\author{
Urs-Beat Brändli \\ Meinrad Abegg \\ Rita Bütler
}

\author{
Eidgenössische Forschungsanstalt für Wald, Schnee und Landschaft $(\mathrm{CH})^{*}$ \\ Eidgenössische Forschungsanstalt für Wald, Schnee und Landschaft $(\mathrm{CH})$ \\ Eidgenössische Forschungsanstalt für Wald, Schnee und Landschaft (CH)
}

\section{Identifying habitat hotspots for saproxylic species using NFI data}

\begin{abstract}
Old wood and deadwood are the most important habitat features for thousands of typical wood-dwelling animal, fungi and plant species. In this paper, we explore the question of whether Swiss National Forest Inventory (NFI) data can be used to identify so-called habitat hotspots in order to promote species nationally and regionally. We therefore used NFI data to analyse old wood, deadwood and habitat trees, which are the three most important habitat indicators for old and deadwood specialists, and developed a structural index based on all three. The results show that national distribution maps of selected saproxylic species differ in how well they correspond with NFI habitat indicators, most probably because field observations are not representative. The indicators were therefore compared with modelled distribution areas of potential hotspots for saproxylic species. It seems that today deadwood in particular is seldom very frequent in those areas where it would most help to maintain biodiversity. Deadwood is especially plentiful in the western edges of the Alps. There are large supplies of old wood in Valais and the Engadine, and hotspots of habitat trees in Ticino, the central Jura and lower Valais. The NFI data on site characteristics, management forms and main forest functions were analysed to find out how much influence these factors have. For example, spruce-silver fir forests contain twice as much deadwood as beech forests ( 32 vs. $16 \mathrm{~m}^{3} / \mathrm{ha}$ ), and old stands in spruce-silver fir forests untended for over 30 years as much as $50 \mathrm{~m}^{3} / \mathrm{ha}$. Deadwood supplies tend to be greater at higher altitudes, on steep slopes and in forests difficult to access, regardless of the forest function. Protection forests tend to contain the most old stands ( $27 \%$ older than 120 years) and deadwood ( $\left.26 \mathrm{~m}^{3} / \mathrm{ha}\right)$, while forests mainly used for recreation and timber production contain $12-13 \mathrm{~m}^{3} /$ ha of deadwood and $17-20 \%$ of old stands. During the past two decades, the amount of old wood and deadwood has greatly increased in forests regardless of their function. The NFI inventories are very suitable for monitoring changes in forest habitats across space and time, and for assessing the influence of site characteristics, forms of management and natural events. Detailed analyses of NFI data can help in deciding on the best nature conservation strategies, for example, the geographical focus for conservation or promotion, the potential of forests that have not been used for a long time or the synergy with protection forests. NFI data should be used more for such purposes in future.
\end{abstract}

Keywords: forest biodiversity, National Forest Inventory, saproxylic species, deadwood, habitat tree doi: $10.3188 /$ szf. 2011.0312

*Zürcherstrasse 111, CH-8903 Birmensdorf, E-Mail urs-beat.braendli@wsl.ch

$\mathrm{D}$ ie Strategie zur Erhaltung und Förderung der Biodiversität im Schweizer Wald erfordert sowohl segregative (z.B. Waldreservate für den Prozessschutz) als auch integrative Massnahmen (z.B. Totholzförderung im Wirtschaftswald) und gezielte Artenförderungsprogramme (Bollmann et al 2009). Dabei bedienen sich Bund und Kantone zur Abklärung von Zustand und Entwicklung der Biodiversität sowie für die Massnahmenplanung und Erfolgskontrolle verschiedener Instrumente, zum Beispiel des Biodiversitätsmonitorings (BDM; Koordinationsstelle Biodiversitäts-Monitoring Schweiz
2009). Was die Qualität der Waldbiotope betrifft, steht das Landesforstinventar (LFI) auf Bundesebene an erster Stelle, und die erhobenen Lebensraumindikatoren sind von bisher unbestrittener Relevanz (Brändli et al 2007a).

So gelten Alt- und Totholz als die wichtigsten Lebensraumaspekte für Tausende typischer waldbewohnender Tier-, Pilz- und Pflanzenarten (saproxylische Arten) ${ }^{1}$, und entsprechende nationale Kon-

\footnotetext{
1 Arten, die während eines Teils ihres Lebenszyklus auf Totholz oder auf andere saproxylische Arten angewiesen sind.
} 
zepte zu deren Förderung wurden auch auf der Basis von LFI-Daten entwickelt (Bütler et al 2006, Bütler et al 2005). Weitere Indikatoren aus dem LFI sind etwa die Struktur der Waldränder, die Dichte der Bestände, die Anzahl der Gehölzarten oder die Anteile an Naturverjüngung und Exoten (Brändli et al 2010). Solche Lebensraumindikatoren werden im Rahmen der Ergebnisberichte LFI (Brändli 2010), der nationalen Umweltberichte (Buwal \& WSL 2005), im Netzwerk Umweltdaten Schweiz von Bund und Kantonen sowie im internationalen Reporting verwendet (Brändli et al 2007a).

Im Interesse einer maximalen Nutzung der LFI-Daten stellt sich die Frage nach der möglichen räumlichen und zeitlichen Auflösung von Aussagen über Waldstrukturen. Wo liegen die Schwerpunkte wertvoller Lebensräume, auch Hotspots genannt, und wie entwickeln sie sich? Die praktische Relevanz liegt dabei etwa in der «Hotspot-Strategie» (Meyer et al 2009, Hobohm 2005, Myers et al 2000), die besagt, dass Massnahmen zur Sicherung der Biodiversität dort ihre grösste Wirkung entfalten können, wo noch mehr oder weniger intakte Zentren vorhanden sind, sowohl bezüglich naturnaher Arten- wie auch Biotopvielfalt. Besonders «wertvolle» Lebensräume sind dabei solche, die sogenannte Urwaldreliktarten beherbergen, die auf urwaldtypische Strukturen und eine durchgängige Habitattradition angewiesen sind (Müller et al 2005, Bussler 2010). Ein ähnlicher Hotspot-Ansatz, jedoch auf Bestandesebene, wurde in Norwegen getestet (Gjerde et al 2007).

Der vorliegende Beitrag geht der Frage nach, ob und mit welchen LFI-Daten aus Stichprobeflächen im 1.4-km×1.4-km-Netz sogenannte Lebensraum-Hotspots für eine effiziente nationale und regionale Artenförderung ermittelt werden können. Betrachtet werden dabei nur jene Lebensraumaspekte, die durch ein Management verändert werden können, also die Waldstrukturen. Dabei liegt der Fokus auf den wichtigsten Lebensraumaspekten typischer Waldarten des Naturwaldes: dem Alt- und Totholz sowie den sogenannten Habitatbäumen, die auch als Trittsteine zwischen Reservaten und Altholzinseln von Bedeutung sind (Meier 2009, Winter \& Möller 2008, Winter et al 2003). Anhand von Verbreitungskarten ausgewählter Schirmarten werden Zusammenhänge mit LFI-Daten untersucht, und mittels LFI-Zweitaufnahmen wird die Aussagekraft einzelner Indikatoren beurteilt. Geeignete Indikatoren werden auf ihre zeitliche und räumliche Entwicklung hin analysiert und auf Zusammenhänge mit der Bewirtschaftung untersucht. Dabei wird auch der Frage nachgegangen, ob sich die Sturmereignisse Vivian und Lothar oder Förderungsstrategien in der Verteilung des Totholzvorrates abzeichnen. Durch die Überlagerung (Kumulation) von Indikatoren werden Hotspots oder Potenzialgebiete für mögliche künftige Massnahmen dargestellt.

\section{Material und Methode}

\section{Erhebung und Analyse der LFI-Daten}

Das aktuelle LFI ist als Stichprobenerhebung auf einem 1.4-km×1.4-km-Netz angelegt. Die Anzahl Waldprobeflächen beträgt rund 6500. Als Datenquellen dienen Luftbilder, terrestrische Erhebungen und Umfragen beim Forstdienst sowie digitalisierte Karten, Modelle (z.B. Vegetationseinheiten) und Erhebungen von Dritten (Areal- und Forststatistik des Bundesamts für Statistik, Klimadaten von Meteo Schweiz).

Auf der sogenannten Interpretationsfläche von $50 \mathrm{~m} \times 50 \mathrm{~m}\left(2500 \mathrm{~m}^{2}\right)$ werden die Luftbilder interpretiert und im Gelände flächenbezogene terrestrische Daten zum Standort und zum Bestand erfasst. Die Baumdaten werden auf zwei konzentrischen Kreisflächen erhoben. Im kleineren Kreis von $200 \mathrm{~m}^{2}$ werden alle Bäume mit einem Brusthöhendurchmesser (BHD) ab 12 cm, im grösseren Kreis von $500 \mathrm{~m}^{2}$ alle Bäume mit einem BHD ab $36 \mathrm{~cm}$ vermessen und beurteilt. Dabei werden alle stehenden und liegenden, lebenden und toten Bäume erfasst. Die hier vorgestellten Ergebnisse basieren auf diesen Daten. Weitere Module des LFI sind die Jungwalderhebung, die Waldrandinventur und seit dem LFI3 das «line-intersect sampling» von liegendem Moderund Totholz ab $7 \mathrm{~cm}$ Durchmesser. Der Arbeitsablauf auf der Probefläche und alle erhobenen Merkmale sind in der Aufnahmeanleitung beschrieben (Keller 2010). Für die Überprüfung der Datenqualität erfolgt auf rund 10\% der Probeflächen eine unabhängige Zweitaufnahme.

Alle statistischen LFI-Ergebnisse werden, zur Vermeidung von Fehlinterpretationen, grundsätzlich zusammen mit den Schätzfehlern publiziert. Zur Visualisierung der räumlichen Verteilung von Merkmalen werden im LFI drei Typen von Karten verwendet: Karten mit regionalen Mittelwerten, Punktkarten der Probeflächen und Karten mit gleitendem Mittelwert. Letztere werden hier eingesetzt, um Hotspots zu einzelnen Lebensraumindikatoren darzustellen. Dabei werden für jeden Rasterpunkt in einer Umgebung von $7 \mathrm{~km} \times 7 \mathrm{~km}$ die Werte der Probeflächen gemittelt. Mittels GIS dargestellt werden diese Mittelwerte, falls mindestens zwei Waldprobeflächen mit Informationen im umliegenden Quadrat liegen, was einem Waldanteil von mindestens $8 \%$ entspricht.

\section{Lebensraumindikatoren}

Lebensgemeinschaften der Alters- und Zerfallsphase von Laubwäldern und von Sonderstandorten im Wald wie Moor-, Bruch-, Trocken- oder Blockschuttwäldern beinhalten zusammengenommen den grössten Teil der waldgebundenen Artenvielfalt (Meyer et al 2009). Als grösstes ökologisches Defizit im gut erschlossenen Wirtschaftswald be- 


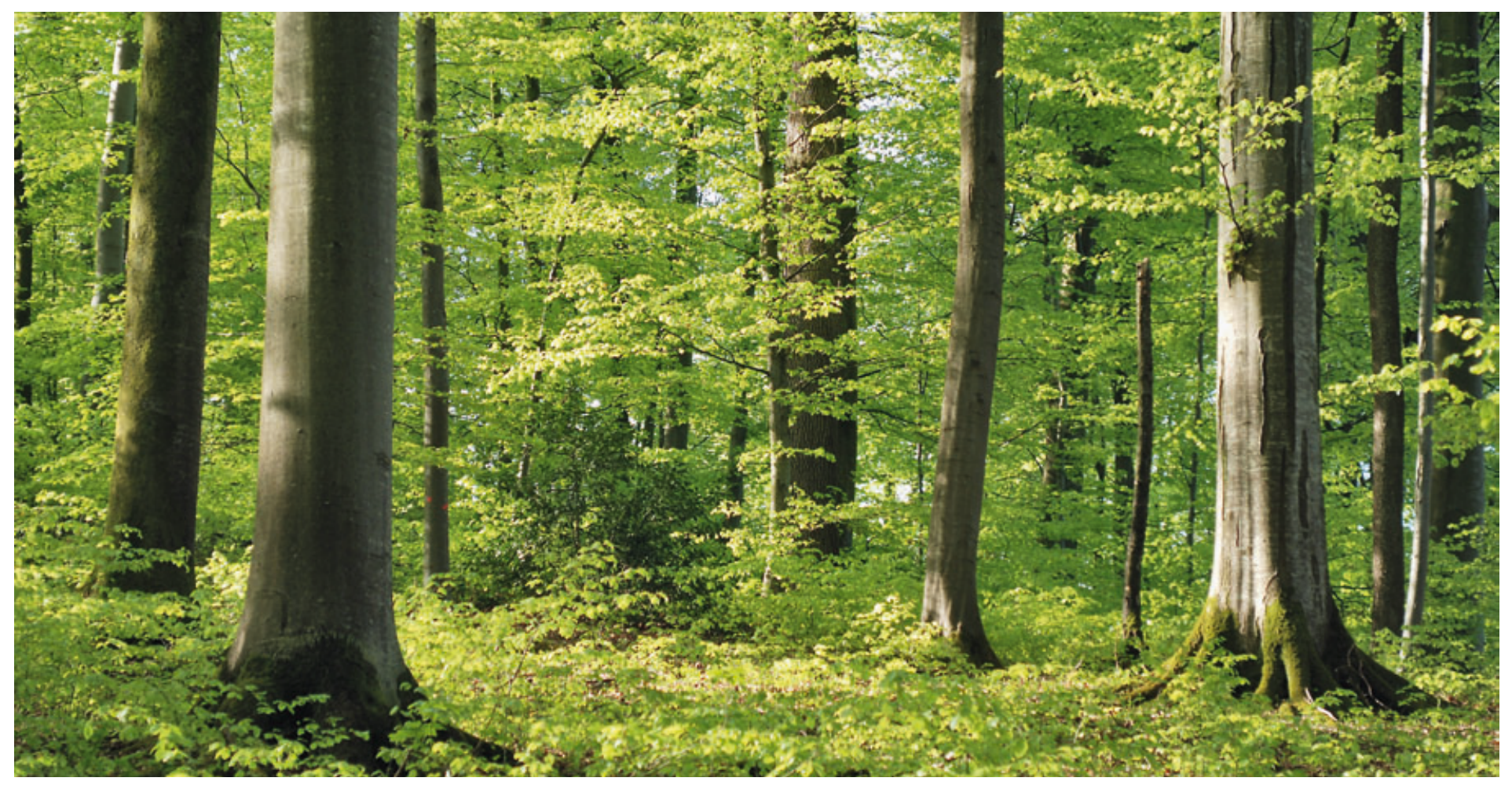

Abb 1 «Urwaldreliktarten» sind auf Habitattradition und damit auf «uralte» Bäume oder Baumbestände angewiesen.

zeichnete der Naturschutz schon vor über 20 Jahren anhand von LFI-Daten den Mangel an Alt- und Totholz (SBN 1989). Eine der Strategien von Bund und Kantonen ist die Förderung von Altholzinseln und Waldreservaten (Projektleitung WAP-CH \& BHPBrugger 2004). Da aber viele xylobionte ${ }^{2}$ Waldarten über eine sehr begrenzte Ausbreitungsfähigkeit verfügen, sind sie und auch andere Waldarten auf eine Vernetzung der Altbestände angewiesen (Winter et al 2003, Müller 2005). Diese Funktion als Trittsteine sollen sogenannte Biotop- oder Habitatbäume erfüllen (Meier 2009, Lachat \& Bütler 2007). Aus diesen Gründen fokussiert die vorliegende Analyse der LFIDaten auf die Indikatoren «Altholz», «Totholz» und «Habitatbaum».

\section{Altholz}

Die stark gefährdeten «Urwaldreliktarten» sind auf Habitattradition und damit auf «uralte» Bäume oder Baumbestände angewiesen (Abbildung 1). Für Flechten, Mollusken und Vögel in Buchenwäldern fanden Moning \& Müller (2009)
Schwellenwerte bei einem Bestandesalter von 100 bis 170 Jahren (submontan) respektive 160 bis 220 Jahre (montan) für eine besonders grosse Artenvielfalt.

Fast alle europäischen nationalen Waldinventuren geben Auskunft über das Bestandesalter. In den meisten Fällen handelt es sich, wie im LFI, zur Hauptsache um Schätzungen. Systematische Bohrspanentnahmen an allen Probebäumen bilden die Ausnahme (Brändli et al 2011). In der Regel liegen nur Angaben für gleichaltrige Bestände vor. In ungleichaltrigen Beständen (z.B. Plenterwälder, Mittelwälder), die in der Schweiz einen Anteil von 22\% ausmachen, stehen aber oftmals zahlreiche alte Bäume, die mit dem Indikator Bestandesalter unberücksichtigt bleiben. Brändli et al (2011) schlagen deshalb in Analogie zu den bekannten Grössen $H_{\text {dom }}$ oder $D_{\text {dom }}$ die Einführung einer neuen Grösse Alter ${ }_{\text {dom }}$ vor. Die Autoren belegen anhand von Daten aus verschiedenen Län-

2 Arten, die zumindest einen Teil ihres Lebens in oder auf Holz leben und sich mindestens teilweise von Holz ernähren.

\begin{tabular}{|c|c|c|c|c|c|c|c|c|}
\hline & Keine Angabe & 0-40 Jahre & 41-80 Jahre & 81-120 Jahre & 121-160 Jahre & $>160$ Jahre & Total & Durchschnitt \\
\hline & $\mathbf{N}$ & $\bar{N}$ & $\bar{N}$ & $\bar{N}$ & $\mathbf{N}$ & $\bar{N}$ & $\bar{N}$ & Jahre \\
\hline Bestandesalter & 230 & 199 & 160 & 190 & 123 & 67 & 969 & 88.4 \\
\hline Alter $_{\text {dom }}$ & 36 & 111 & 232 & 332 & 175 & 83 & 969 & 99.4 \\
\hline Bestandesalter & 24 & 21 & 17 & 20 & 13 & 7 & 100 & \\
\hline Alter dom $_{\text {d }}$ & 4 & 11 & 24 & 34 & 18 & 9 & 100 & \\
\hline
\end{tabular}

Tab 1 Vergleich des Bestandesalters und des vorherrschenden Baumalters (Alter dom) auf Probeflächen der Inventur LFI4 (Datenstand 19.8.2010). N: Anzahl Probeflächen. 


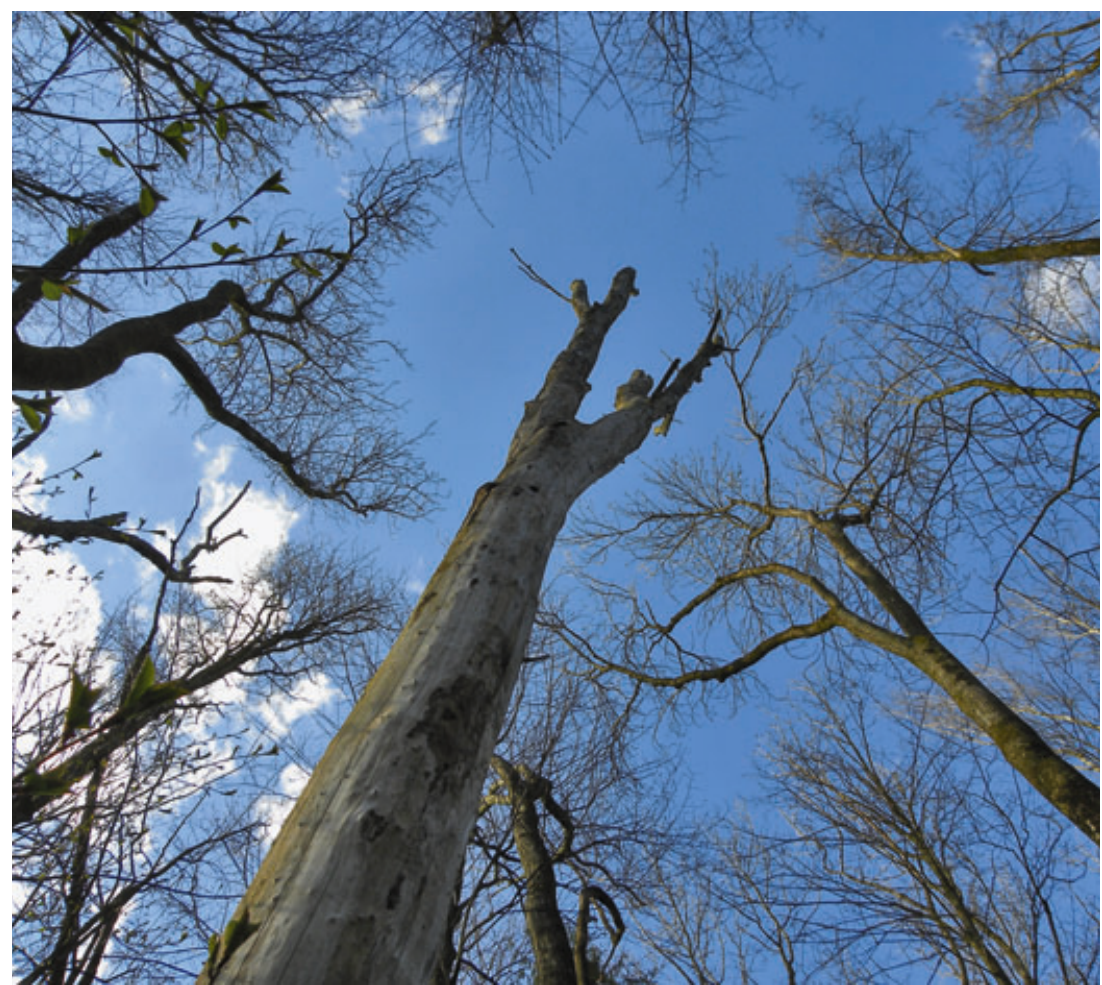

Abb 2 Grosse tote Bäume sind ein wesentliches Merkmal in der zweiten Lebenshälfte von Naturwäldern.

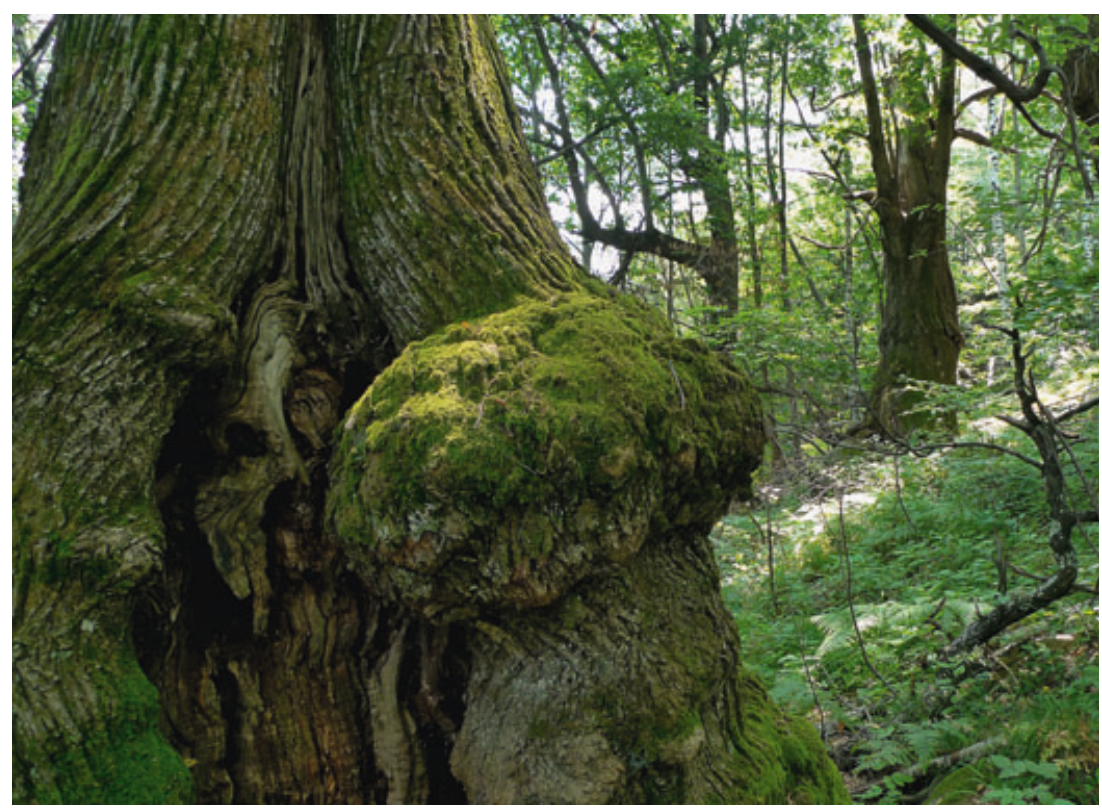

Abb 3 Habitatbäume weisen in der Regel Strukturmerkmale wie Dürräste, Risse, Spechtlöcher oder Rindenverletzungen auf.

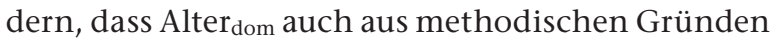
viel geeigneter ist für internationale Vergleiche als das herkömmliche Bestandesalter.

Das vorherrschende Baumalter (Alter ${ }_{\mathrm{dom}}$ ) ist definiert als das durchschnittliche Alter der 100 dicksten Bäume pro Hektare. Damit diese Grösse berechnet werden kann, wird im laufenden LFI4 seit 2009 erstmals auch das Alter jedes Probebaumes geschätzt und erfasst. Die derzeit vorhandenen Daten reichen aber für eine Hotspot-Analyse noch nicht aus. Sie erlauben jedoch erste Vergleiche mit dem Bestandesalter. Das Bestandesalter konnte nur für $76 \%$ der zugänglichen Probeflächen bestimmt werden, das Alter $_{\text {dom }}$ hingegen für $96 \%$, weil damit auch ungleichaltrige Bestände erfasst werden (Tabelle 1). Und weil die vorherrschenden Bäume in ungleichaltrigen Beständen oftmals alt sind (z.B. Gebirgsplenterwälder), sind das Durchschnittsalter und der Anteil Altholzbestände beim Alter $_{\text {dom }}$ erheblich höher als beim Bestandesalter.

\section{Totholz}

Grosse tote Bäume sind ein wesentliches Merkmal in der zweiten Lebenshälfte von Naturwäldern (Abbildung 2). Im Gegensatz zu traditionell wirtschaftlich orientierten Waldinventuren wurden bereits im LFI1 auch tote stehende und liegende Probebäume ab $12 \mathrm{~cm}$ BHD erfasst, allerdings nur, sofern das Holz noch mindestens als Brennholz hätte verwertet werden können. Im LFI2 wurden dann alle toten Bäume mit erkennbarer Baumart als Totholzvorrat erfasst. Im LFI3 kamen noch tote Probebäume ohne erkennbare Baumart hinzu und wurden zusammen mit dem Totholzvorrat als sogenanntes Totholzvolumen ausgewiesen (Brändli 2010). Für das Totholzvolumen wurden auch erstmals qualitative Aspekte wie Holzfestigkeit, Rindenbedeckung oder das Vorkommen von Spechtlöchern und Holzpilzen erhoben. Für Zeitreihenvergleiche LFI1-LFI2-LFI3 sind Aussagen zum Totholzvorrat differenziert nach stehend/liegend, Baumarten und Durchmesser möglich. Es gilt zu beachten, dass die Totholzvorräte im LFI1 wegen unterschiedlicher Definition gegenüber LFI2 und LFI3 um etwa einen Drittel unterschätzt werden. Als Einheit wird in der Regel $\mathrm{m}^{3} /$ ha verwendet, da diese für das Vorkommen von Organismen und damit für die (inter)nationale Berichterstattung massgebend ist. Der Totholzvorrat wird berechnet als Schaftholz in Rinde inklusive Stock, ungeachtet allfälliger Schaftbrüche. Stöcke von geernteten Bäumen und Holzernteresten sind nicht enthalten.

\section{Habitatbaum}

Das vorliegende Habitatbaum-Modell basiert auf der Arbeit von Meier (2009, siehe auch Niedermann-Meier et al 2010) und bezieht sich auf lebende Bäume mit Habitatstrukturen (Abbildung 3). Meier hat aufgrund von Experteninterviews und Literaturangaben die verschiedenen Habitatstrukturen sowie die Baumart und den Baumdurchmesser nach ihrer Bedeutung für die fünf Organismengruppen Fledermäuse, Insekten, Vögel, Flechten und Pilze gewichtet. Im vorliegenden LFI-Modell werden die Mittelwerte der fünf Gruppen verwendet. Eingangsgrössen für das Strukturmodell sind die im LFI erfassten Baumschäden und Bemerkungen. Damit wurde gemäss Tabelle 2 für jeden lebenden Probebaum der aufsummierte Habitatwert berechnet. Diese Werte wurden anschliessend als Habitatindex pro Probefläche beziehungsweise pro Hektare hochgerechnet. 


\begin{tabular}{|c|c|c|c|}
\hline Kriterium & Ausprägung & Habitatwert & Gewichtung \\
\hline \multicolumn{4}{|l|}{ Strukturmerkmale } \\
\hline \multirow[t]{2}{*}{ Dürrastanteil > $20 \%$ der Astmasse } & Nadelbaum & 0 & 3 \\
\hline & Laubbaum & 2.4 & 3 \\
\hline Gipfel dürr & & 2.4 & 3 \\
\hline Kronen-, Stammbruch & & 3 & 3 \\
\hline \multirow[t]{2}{*}{ Spechtlöcher und -ringe } & Stammfuss & 1.8 & 3 \\
\hline & Stamm, Schaft, Krone & 2 & 3 \\
\hline \multirow[t]{2}{*}{ Holzkörper frei 0 bis 4 Handflächen } & Stammfuss & 1.4 & 3 \\
\hline & Stamm, Schaft, Krone & 1.8 & 3 \\
\hline \multirow{2}{*}{ Holzkörper frei > 4 Handflächen } & Stammfuss & 2 & 3 \\
\hline & Stamm, Schaft, Krone & 2.2 & 3 \\
\hline \multirow[t]{2}{*}{ Riss $\leq 1 \mathrm{~m}$} & Stammfuss & 2 & 3 \\
\hline & Stamm, Schaft, Krone & 2.2 & 3 \\
\hline \multirow[t]{2}{*}{ Riss $>1 \mathrm{~m}$} & Stammfuss & 2.4 & 3 \\
\hline & Stamm, Schaft, Krone & 2.6 & 3 \\
\hline Capitozzi ( $\geq 3$ Stammachsen) & unter 7.0 m Höhe & 2.8 & 3 \\
\hline Zwiesel (zwei Stammachsen) & in 1.3 bis $9.0 \mathrm{~m}$ Höhe & 1.4 & 3 \\
\hline Liegend oder Stockausschlag & & 0.8 & 3 \\
\hline Krebs $>1 / 2$ Stamm oder Rindennekrosen & & 2.6 & 3 \\
\hline Pilzfruchtkörper & & 2 & 3 \\
\hline \multirow[t]{6}{*}{ Baumart } & Föhren (Pinus spp.) & 2 & 1 \\
\hline & Lärchen (Larix spp.) & 1 & 1 \\
\hline & Fichte, Tanne, übrige Nadelbäume & 0 & 1 \\
\hline & Eichen (Quercus spp.) & 4 & 1 \\
\hline & $\begin{array}{l}\text { Erlen (Alnus spp.), Pappeln (Populus spp.), } \\
\text { Kirschbaum (Prunus sp.) }\end{array}$ & 2 & 1 \\
\hline & Buche (Fagus sp.), übrige Laubbäume & 1 & 1 \\
\hline \multirow[t]{5}{*}{ Brusthöhendurchmesser (BHD) } & $<40 \mathrm{~cm}$ & 0 & 1 \\
\hline & $40-59 \mathrm{~cm}$ & 1 & 1 \\
\hline & $60-79 \mathrm{~cm}$ & 2 & 1 \\
\hline & $80-99 \mathrm{~cm}$ & 3 & 1 \\
\hline & $\geq 100 \mathrm{~cm}$ & 4 & 1 \\
\hline
\end{tabular}

Tab 2 Bewertung und Gewichtung von Strukturmerkmalen, Baumart und Durchmesser im LFI-Modell Habitatbaum.
Im LFI3 liegen die erreichten Werte im Bereich von 0 bis 30 für den Habitatwert von Probebäumen respektive 0 bis 14938 für den Habitatindex pro Hektare. Der aufsummierte Habitatwert sämtlicher LFI3Probebäume resultiert zu $66 \%$ aus den gewichteten Strukturmerkmalen, zu 25\% aus der Baumart und zu 9\% aus dem BHD.

\section{Aussagekraft von LFI-Lebensraumindikatoren}

Die Aussagekraft von Indikatoren ist im Wesentlichen von der Datenqualität und der Relevanz der Daten abhängig. Die Qualität und die Reproduzierbarkeit der LFI-Daten lassen sich mittels statistischer Analysen von Erst- und unabhängigen Zweitaufnahmen beurteilen. Auf dieser Basis können die Genauigkeiten von Messungen (z.B. BHD), aber auch die Beobachtungswahrscheinlichkeiten etwa von Habitatstrukturen ermittelt werden. Daraus lässt sich die effektive Häufigkeit von Phänomenen abschätzen, und ihre Wertung in Habitatmodellen kann entsprechend korrigiert beziehungsweise gewichtet wer- den. Am Beispiel des Schadenbildes «Holzkörper freigelegt» zeigt sich, dass selbst grosse und gut einsehbare Schäden nur zu maximal 50\% aufgenommen werden (Tabelle 3). Aus diesem Grund wurden die im LFI erfassten Strukturmerkmale gegenüber Baumart und BHD dreifach gewichtet (Tabelle 2).

Die Relevanz von Lebensraumindikatoren, das heisst der Nachweis ihrer Bedeutung für das Vorkommen einer Art oder einer Artengruppe, lässt sich am Beispiel von Stichprobedaten nur begrenzt zeigen, selbst wenn gewisse Daten wie im Falle des Biodiversitätsmonitorings Schweiz (BDM) und des LFI auf gemeinsamen Probeflächen erhoben werden. Vermutlich sind die Flächen dafür zu klein, und wichtige Lebensraumvariablen fehlen oder haben nicht die nötige Auflösung. Aus der Literatur sind jedoch solche Zusammenhänge für viele Arten- und Artengruppen klar belegt (Brändli et al 2007b). Somit kann die Gegenüberstellung von Verbreitungs- und Indikatorkarten für das Arten- und Umweltmanagement durchaus relevant sein. 


\begin{tabular}{|l|c|c|c|}
\multirow{2}{*}{ Schadenort } & \multicolumn{3}{|c|}{ Schadengrösse } \\
\cline { 2 - 4 } & $<1$ Handfläche & $1-4$ Handflächen & $>$ 4 Handflächen \\
\hline Krone & $*$ & $*$ & $*$ \\
\hline Schaft & 0.19 & $*$ & 0.37 \\
\hline Stamm & 0.35 & 0.37 & 0.48 \\
\hline Stammanlauf & 0.35 & 0.43 & 0.48
\end{tabular}

Tab 3 Aufnahmewahrscheinlichkeit des Schadens «Holzkörper freigelegt» (Rindenschürfung). * zu wenige Beobachtungen für gesicherte Aussagen.

\section{Messungen versus Schätzungen und Beobachtungen}

Geht es um den Nachweis von langsamen Veränderungen, wie dies beim Lebensraum Wald der Fall ist, so sind Messungen und davon abgeleitete Grössen (Totholzvorrat) offensichtlich geeigneter als gutachtliche Schätzungen und Beobachtungen (Bestandesalter, Strukturmerkmale). So resultiert zum Beispiel aus dem Vergleich von Erst- und Zweitaufnahmen des LFI3 beim gemessenen BHD eine Standardabweichung von $1.5 \mathrm{~cm}$ (5\% des mittleren BHD), beim geschätzten Bestandesalter eine solche von 34 Jahren (36\% des Mittelwerts). Deshalb werden BHD und $\mathrm{D}_{\text {dom }}$ oft als Proxy-Variablen für Habitatstrukturen und Bestandesalter vorgeschlagen. Am Beispiel von Daten des schwedischen Landesforstinventars, bei dem das Alter aller Probebäume ermittelt wird, zeigten Brändli et al (2011), dass der BHD nur 33\% der Variation des Baumalters erklärt.

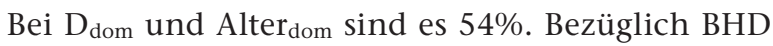
und des aufsummierten Habitatwerts der Strukturmerkmale eines LFI-Probebaumes konnte kein $\mathrm{Zu}$ sammenhang nachgewiesen werden $\left(\mathrm{R}^{2}<0.1\right)$, wobei die dicksten Bäume ( $\geq 52 \mathrm{~cm}$ BHD) mit 26\% sogar weniger häufig Strukturmerkmale aufweisen als die dünnsten (12-15 cm BDH) mit 33\%. Auch die logische Hypothese, dass Habitatstrukturen mit zunehmendem Baumalter ebenfalls zunehmen, konnte anhand von LFI4-Daten nicht bestätigt werden. Dies mag teilweise dadurch bedingt sein, dass im bewirtschafteten Wald die Bäume ohne Strukturmerkmale gefördert und stehen gelassen werden. Dementsprechend werden im LFI keine Proxy-Variablen für Bestandesalter und Strukturmerkmale verwendet.

\section{Ergebnisse}

Artverbreitungen und Lebensraum-Hotspots Für das Vorkommen von Alt- und Totholzspezialisten sind in der Regel mehrere Lebensraumfaktoren von Bedeutung. Es ist deshalb nicht zu erwarten, dass sich die Verbreitung einzelner Tier- und Pflanzenarten direkt mit den Hotspots eines einzigen Lebensraumindikators deckt, zumal die Artenverbreitungen nicht selten unsystematisch erhoben und gemeldet werden. Systematische, repräsentative
Stichprobenerhebungen wie das LFI oder BDM bilden die Ausnahme. Am Beispiel einiger typischer Waldarten sind aber doch Ähnlichkeiten in der räumlichen Verteilung von Arten und Lebensraumindikatoren zu erkennen (Abbildungen 4 und 5).

Der Wärme liebende, seltene Grosse Eichenbock (Cerambyx cerdo) gilt als Reliktart von Eichenurwäldern und Hartholzauen in der Zerfallsphase. Er besiedelt durchfeuchtete Stämme von sonnenexponierten, einzeln stehenden kränkelnden Eichen, seltener Buchen, in lichten Beständen. Sein Vorkommen im Rhonetal und in der Südschweiz zeigt grosse Ähnlichkeit mit den Hotspots des Eichenvorrates gemäss LFI (Abbildungen 4a und 4b). Im östlichen Jura und Mittelland mit den grössten Eichenvorräten liegen dagegen zum Grossen Eichenbock kaum Fundmeldungen vor. Möglicherweise sind diese sehr unvollständig. Vielleicht sind die Bestockungen zu dicht, und es mangelt an kränkelnden Eichen. Jedenfalls erscheinen besonders Wälder der Kantone Aargau, Zürich, Thurgau, Schaffhausen und Waadt als vom Eichenbock bisher unbesiedelte potenzielle Förderungsgebiete.

Die Echte Lungenflechte (Lobaria pulmonaria), eine typische Flaggschiffart für Bewohner alter Wälder, lebt als sogenannter Epiphyt auf der Rinde alter Bäume, meist an Buche und Ahorn. Weil die empfindliche Flechte im Wesentlichen auf niederschlagsreiche, milde bis kühle Lagen beschränkt ist, findet man sie hauptsächlich in ungestörten, naturnahen (Berg-)Wäldern, die niemals völlig abgeholzt wurden. Die aktuelle Verbreitung (Abbildung 4c) zeigt denn auch Ähnlichkeiten mit dem Vorkommen alter Laubholzbestände (Abbildung 4d). Nur im Zürcher Oberland und im zentralen Jura wurde die Lungenflechte trotz günstigen Indikatorverhältnissen praktisch nicht registriert.

Der Dreizehenspecht (Picoides tridactylus) ist eine Charakterart des Nadelwaldgürtels oberhalb von 1200 m ü.M. Er bevorzugt naturnahe Bestände mit viel stehendem Fichtentotholz, wo er sich von Insekten in absterbenden oder frisch toten Bäumen ernährt. Gemäss Brutvogelatlas (Schmid et al 1998) hat der Dreizehenspecht zwischen den Erhebungen 1972-76 und 1993-96 sein Areal um über 30\% ausgedehnt, insbesondere in der Westschweiz (Abbildung 5a). Zwischen LFI1 (1983-85) und LFI2 (199395) hat auch der Vorrat an stehendem Fichtentotholz erheblich zugenommen, zum Teil als Folge des Orkans Vivian (Abbildung 5b). Gebietsweise deckt sich diese Zunahme mit der Arealausdehnung des Dreizehenspechtes, z.B. im Waadtländer Jura. Aus dem Neuenburger Jura, dessen Wälder mit rund $3 \mathrm{~m}^{3} /$ ha praktisch kein Totholz aufweisen (Abbildung 6b), wurde der Dreizehenspecht dagegen nicht gemeldet.

Die Bechsteinfledermaus (Myotis bechsteinii) ist eine der wenigen mitteleuropäischen Fledermausarten, die fast ausschliesslich den Wald als Lebens- 
a) Grosser Eichenbock (Cerambyx cerdo)

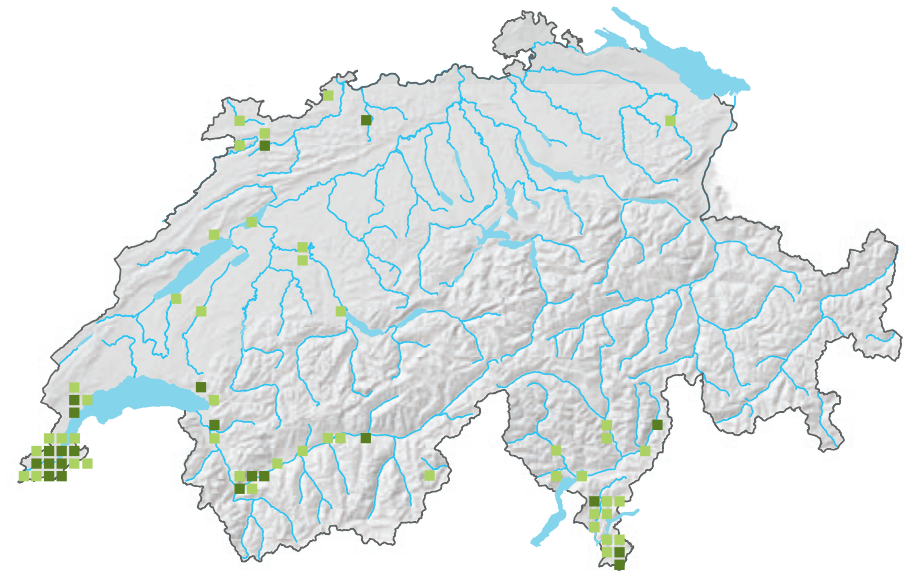

$\square$ vor $2000 \quad \square$ ab 2000

c) Echte Lungenflechte (Lobaria pulmonaria)

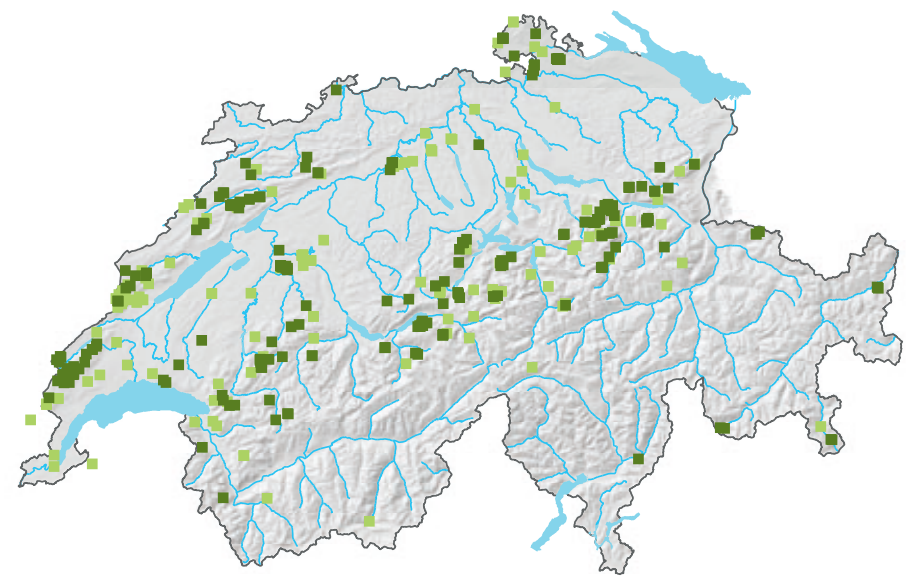

$\square$ vor $1989 \quad \square$ ab 1989 b) Eichenvorrat

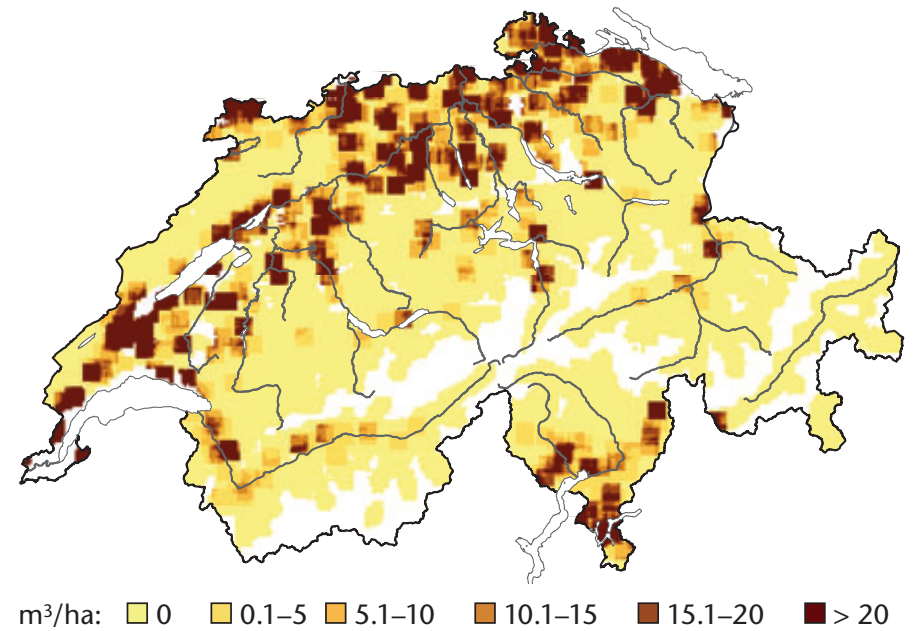

d) Laubholzaltbestände

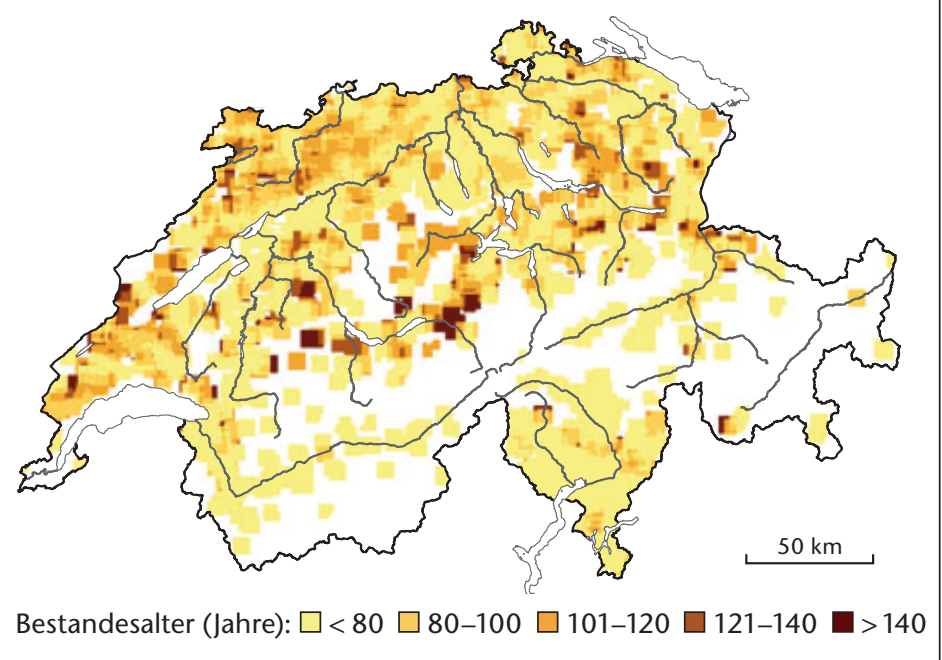

Abb 4 Verbreitung und Vorkommen ausgewählter Arten und Lebensraumindikatoren. a) Grosser Eichenbock (Cerambyx cerdo) vor und ab 2000 (Auszug der Verbreitungskarte im 5-km×5-km-Raster aus dem Kartenserver des Centre Suisse de Cartographie de la Faune, Neuchâtel, Januar 2011), b) Eichenvorrat, c) Echte Lungenflechte (Lobaria pulmonaria) vor 1989 und ab 1989 (Stofer et al 2011), d) Laubholzaltbestände.

raum nutzen. Sie jagt bevorzugt in Buchenaltbeständen und schläft tagsüber am liebsten in Baumhöhlen, aber auch unter abstehenden Baumrinden. Die Verbreitungskarte des Centre Suisse de Cartographie de la Faune (CSCF) in Abbildung 5c zeigt aber kaum Ähnlichkeiten mit dem Vorkommen von Laubholzaltbeständen (Abbildung 4d), Habitatbäumen (Abbildung 5d) oder toten Bäumen mit Spechtlöchern (nicht dargestellt). Vielmehr zeigt sich eine Hauptverbreitung in der Westschweiz in Gebieten mit auffallend wenig Totholz und Spechtlöchern. Die räumliche Nähe der Fledermaus-Hotspots zum CSCF in Neuenburg mag Zufall sein.

\section{Strukturindex}

Altholz, Totholz und Habitatbäume sind die Schlüsselindikatoren für das Vorkommen typischer Waldspezialisten und zeigen unterschiedliche Hotspots (Abbildung 6). Jene der Altbestände liegen beispielsweise im Engadin und im Oberwallis. Die Tot- holzvorräte sind besonders hoch in den westlichen Randalpen, einem Schwerpunkt des Orkanes Lothar. Und die Habitatbäume haben grosse Hotspots im Tessin, im zentralen Jura und im Unterwallis. In der gleichwertigen Kumulation der drei Indikatoren zeigen sich die aktuellen Lebensraum-Hotspots für Altund Totholzarten. Dazu wurden die Wertebereiche der drei Indikatoren je in fünf Quantile aufgeteilt. Werte im ersten Quantil erhielten den Wert 0, solche im fünften den Wert 4, was in der Summe für den sogenannten Strukturindex einen Wertebereich von 0 bis 12 ergibt.

Bütler et al (2006) haben anhand von ökologischen Variablen die potenziellen Verbreitungsgebiete von 55 saproxylischen Arten modelliert. Im Vergleich der kumulierten potenziellen Areale dieser Arten (Abbildung 7) mit dem LFI-Strukturindex (Abbildung 6d) zeigt sich, dass die Wälder in den potenziellen Hotspots der saproxylischen Arten vielfach relativ hohe Strukturindices aufweisen. Un- 


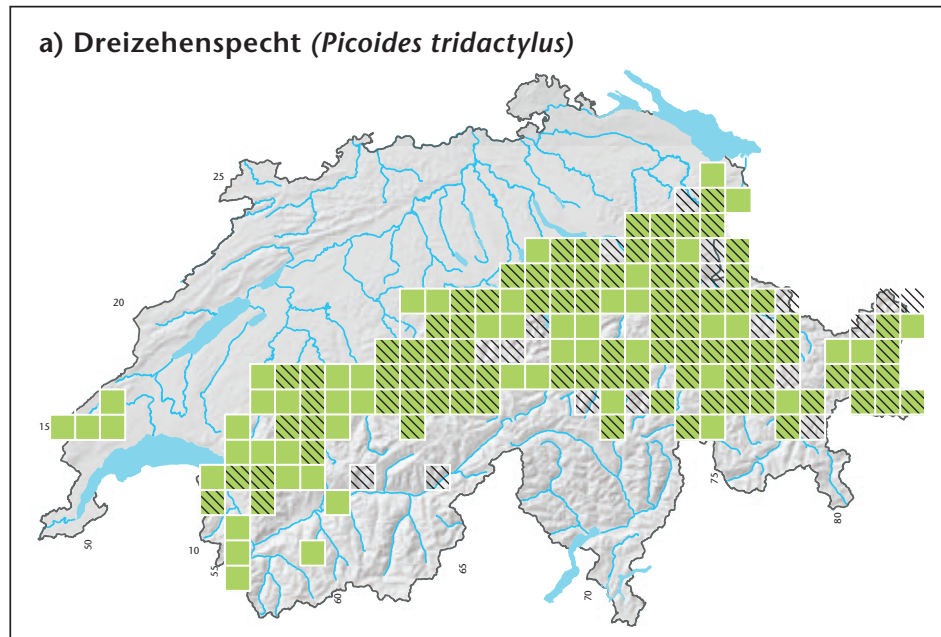

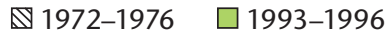

c) Bechsteinfledermaus (Myotis bechsteinii)

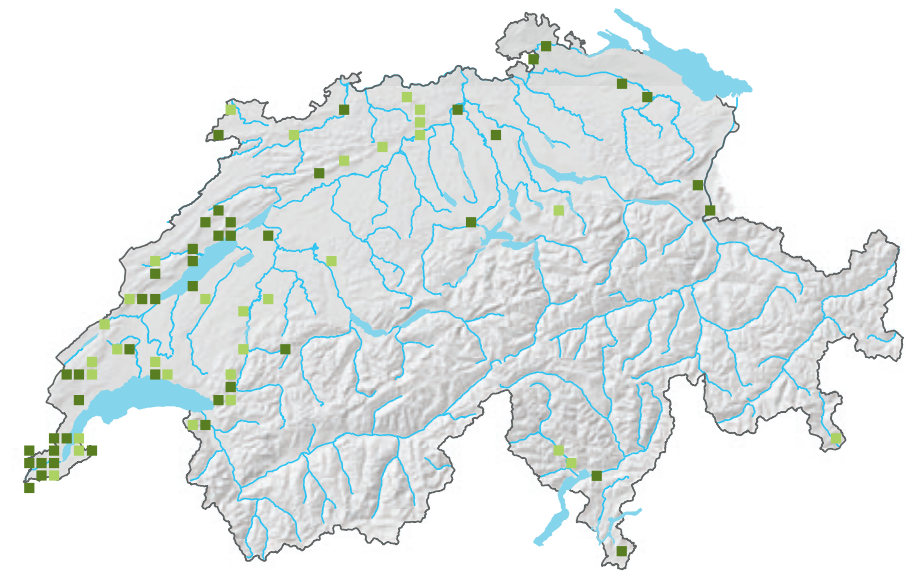

$\square$ vor $2000 \quad \square$ ab 2000 b) Veränderung des Vorrats an stehendem Fichtentotholz

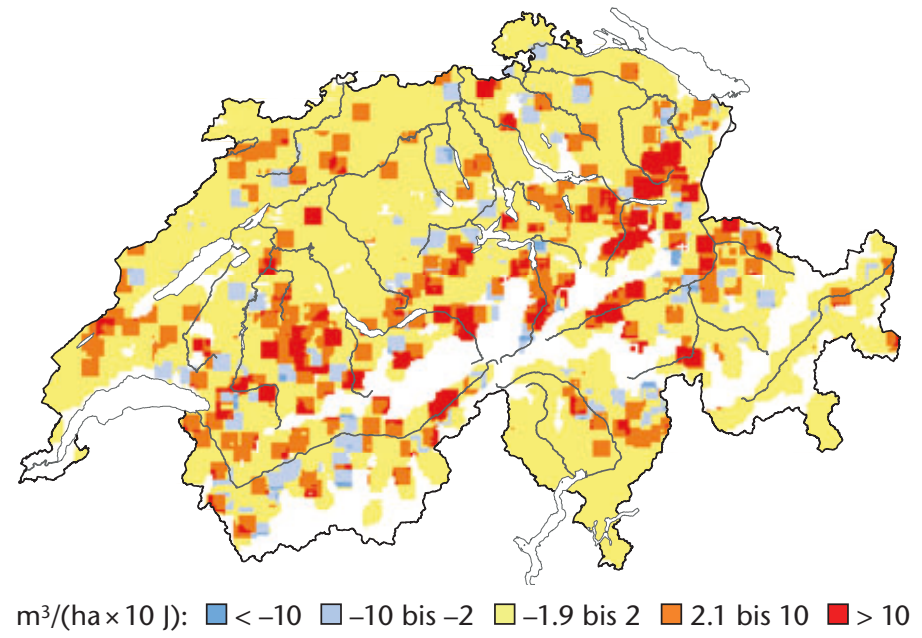

d) Habitatbaumindex

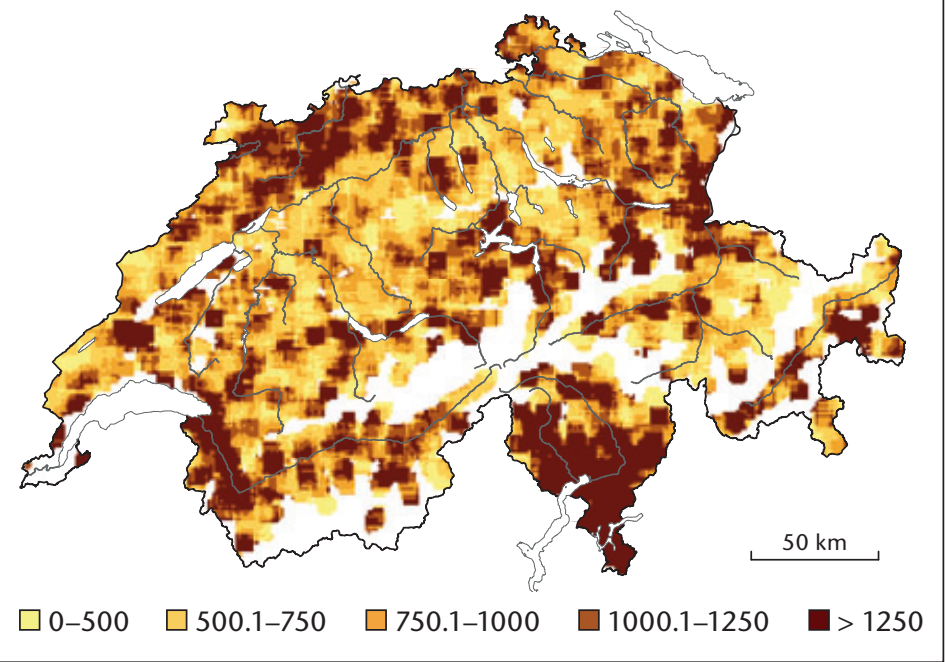

Abb 5 Verbreitung und Vorkommen ausgewählter Arten und Lebensraumindikatoren. a) Dreizehenspecht (Picoides tridactylus) 1972/76 und 1993/96 (Schmid et al 1998), b) Veränderung des Vorrates an stehendem Fichtentotholz 1983/85 bis 1993/95, c) Bechsteinfledermaus (Myotis bechsteinii) vor und ab 2000 (Auszug der Verbreitungskarte im 5-km×5-km-Raster aus dem Kartenserver des Centre Suisse de Cartographie de la Faune, Neuchâtel, Januar 2011; Datengrundlage: Centre de coordination ouest pour l'étude et la protection des chauves-souris und Koordinationsstelle Ost für Fledermausschutz),

d) Habitatbaumindex.

günstig erscheint die Situation aber weitgehend im Mittelland und westlichen Jura. Insbesondere liegt der grösste Teil des Totholzvorrates (Abbildung 6b) ausserhalb der potenziellen «saproxylischen Hotspots». Selbst nach dem Orkan Lothar besteht in vielen Jura- und Mittellandwäldern noch immer ein geringes Totholzangebot.

\section{Totholzqualität}

Neben der räumlichen Verteilung sind beim Totholz auch qualitative Aspekte von Bedeutung. Dicken Dürrständern wird eine grössere ökologische Bedeutung beigemessen als dünnem liegendem Totholz. Analysen der LFI-Daten zeigen, dass dickes und stehendes Totholz ähnlich verteilt ist wie der Totholzvorrat insgesamt. Je nach Höhenlage und Region machen Bäume über $30 \mathrm{~cm}$ BHD rund 60 bis $80 \%$ und stehende Bäume rund 50 bis 70\% des Totholzvorrates aus. Das heisst, schon anhand des gesamten Totholzvorrates kann in der Regel ohne Zusatz- erhebungen auf den Anteil an dickem und stehendem Totholz geschlossen werden. Ähnliches gilt auch für den Abbauzustand des Totholzes.

Einfluss von Standort und Waldmanagement Wie weit sind unsere Wälder vom natürlichen Zustand entfernt? Unter welchen Bedingungen finden sich die höchsten Indikatorwerte? Am Beispiel des Totholzvolumens wird ersichtlich, dass TannenFichten-Wälder heute rund doppelt so viel Totholz aufweisen wie Buchenwälder (Tabelle 4). Frisch genutzte Bestände liegen bezüglich Totholzvorrat unter dem Durchschnitt. Demgegenüber weisen während langer Zeit nicht mehr genutzte Altbestände die höchsten Werte auf, beispielsweise $34 \mathrm{~m}^{3} / \mathrm{ha}$ solche im Areal der Buchenwälder. Dieser Wert ist aber nur halb so gross wie die $70 \mathrm{~m}^{3} / \mathrm{ha}$, die im ukrainischen Buchenurwald Uholka ermittelt wurden (Brändli \& Dowhanytsch 2003). In lange nicht genutzten Altbeständen ist auch der Anteil des abge- 


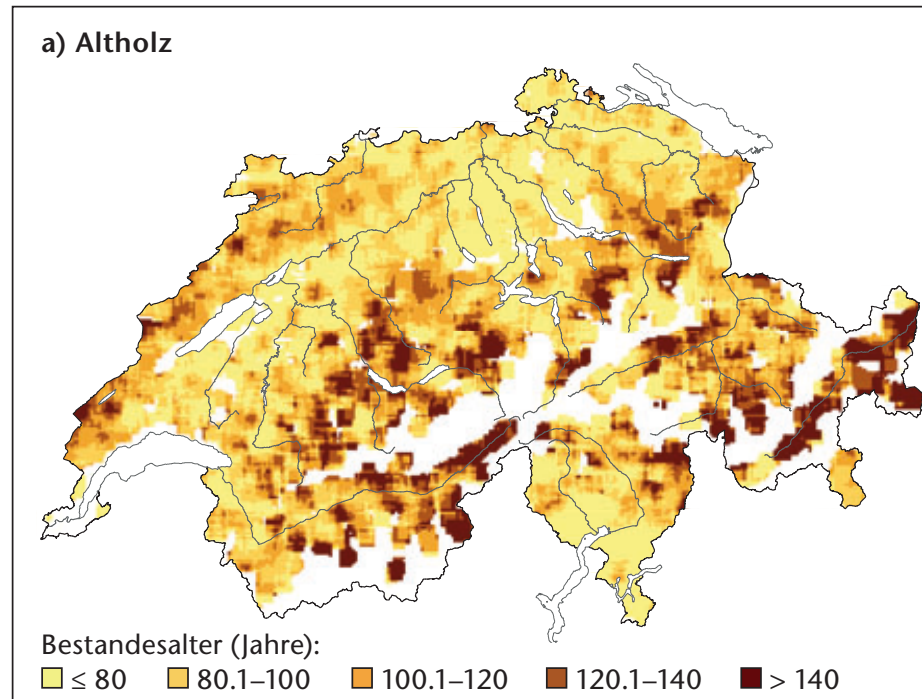

b) Totholzvorrat

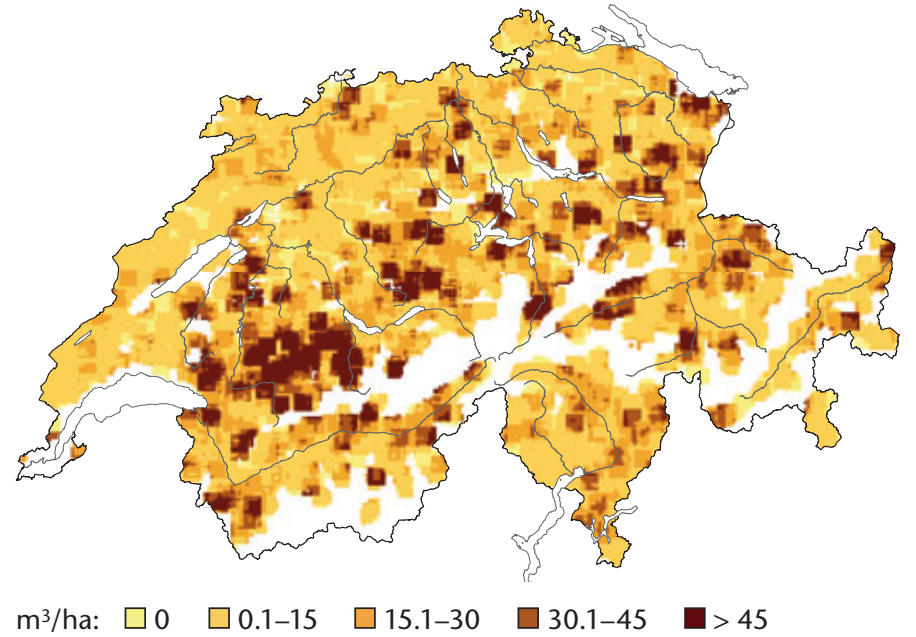

c) Habitatbaumindex

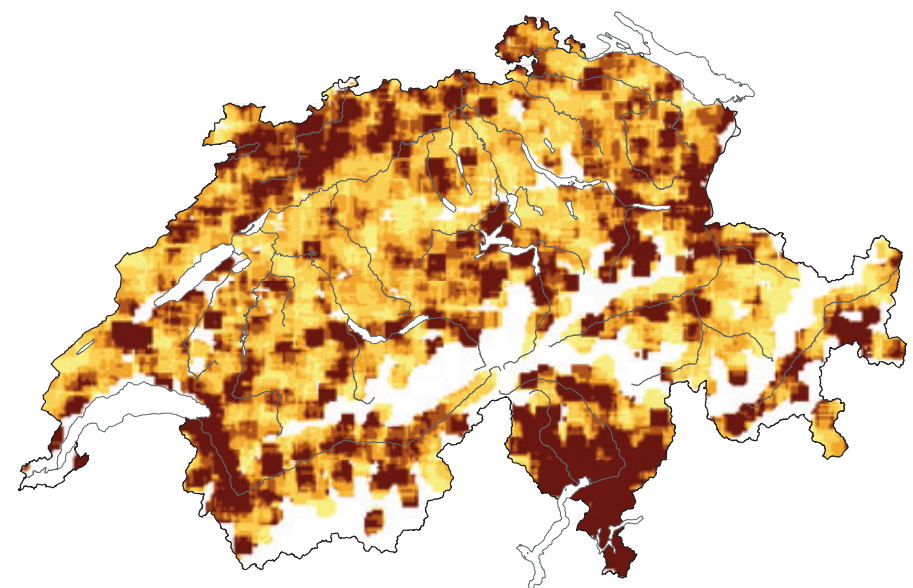

d) Strukturindex

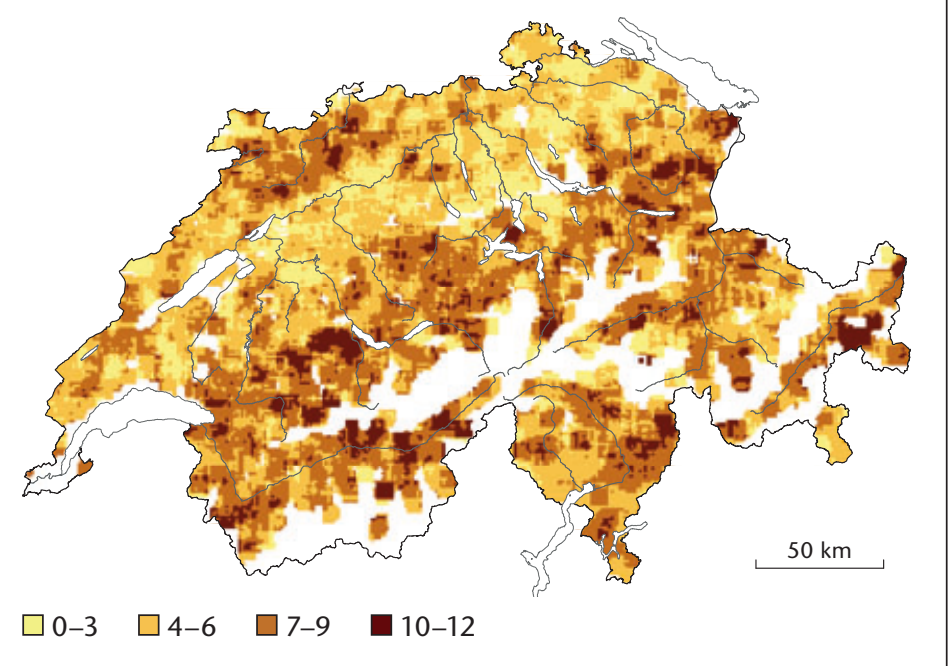

$\square 0-500 \quad \square 500.1-750 \quad \square 750.1-1000$

1000.1-1250

$>1250$

Abb 6 Schwerpunkte (Hotspots) der Lebensraumindikatoren a) Altholz, b) Totholz und c) Habitatbaum sowie Synthese (Wertekumulation) dieser Indikatoren im Strukturindex (d).

bauten Totholzes mit 33\% höher als in relativ frisch genutzten Beständen mit rund 20\%.

Die Stärken des LFI liegen in der objektiven Darstellung von Veränderungen im Schweizer Wald. So haben sich die drei Lebensraumindikatoren «Totholz», «Altholz» und «Habitatbäume» in den zwei Jahrzehnten seit dem LFI1 eindeutig und signifikant positiv entwickelt (Tabelle 5). Der Totholzvorrat hat sich innert elf Jahren fast verdoppelt. Diese Trends zeigen sich gleichermassen im Wirtschafts- und Schutzwald wie auch in Wäldern mit prioritären Naturschutzzielen, also unabhängig vom Hauptziel der Bewirtschaftung, der sogenannten Vorrangfunktion. Am grössten ist der Anteil hochwertiger Habitatbäume im Naturschutzwald, während die Schutzwälder am meisten Altbestände und den höchsten Totholzvorrat aufweisen. Im öffentlichen Wirtschaftswald ist der Totholzvorrat signifikant kleiner als im privaten (Tabelle 6).

Unabhängig von der Waldfunktion sind die Totholzvorräte in höheren Lagen, in steilerem und damit schlechter befahrbarem Gelände sowie in schlechter erschlossenen Wäldern deutlich höher als im übrigen Gebiet (Tabelle 6), wie schon Böhl \& Brändli (2007) zeigen konnten. Das heisst, je höher der Holzernteaufwand ist, desto eher wird Totholz im Wald belassen. Auffallend sind die Verhältnisse im Schutzwald, wo selbst gut erreichbare und leichter zu bewirtschaftende Bestände relativ hohe Totholzvorräte aufweisen. Dies überrascht wenig, sind doch bewusst liegen gelassene tote Bäume als temporäre Schutzverbauungen Teil des Schutzwaldkonzeptes. Dass im frisch genutzten Holzproduktionswald die Totholzvorräte kaum geringer sind als im seit über 20 Jahren nicht genutzten Holzproduktionswald, dürfte auf die Orkane Vivian und Lothar zurückzuführen sein: Viele Nutzungen im Wirtschaftswald waren Zwangsnutzungen, bei denen relativ viel Totholz im Wald belassen wurde. Unabhängig von der Waldfunktion sind die Totholzvorräte in unmittelbarer Strassennähe am geringsten, dort also, wo Totholz am leichtesten erreichbar ist, aber ebenfalls für Passanten am ehesten zur Gefahr werden kann und auch deshalb vermehrt entfernt wird. 


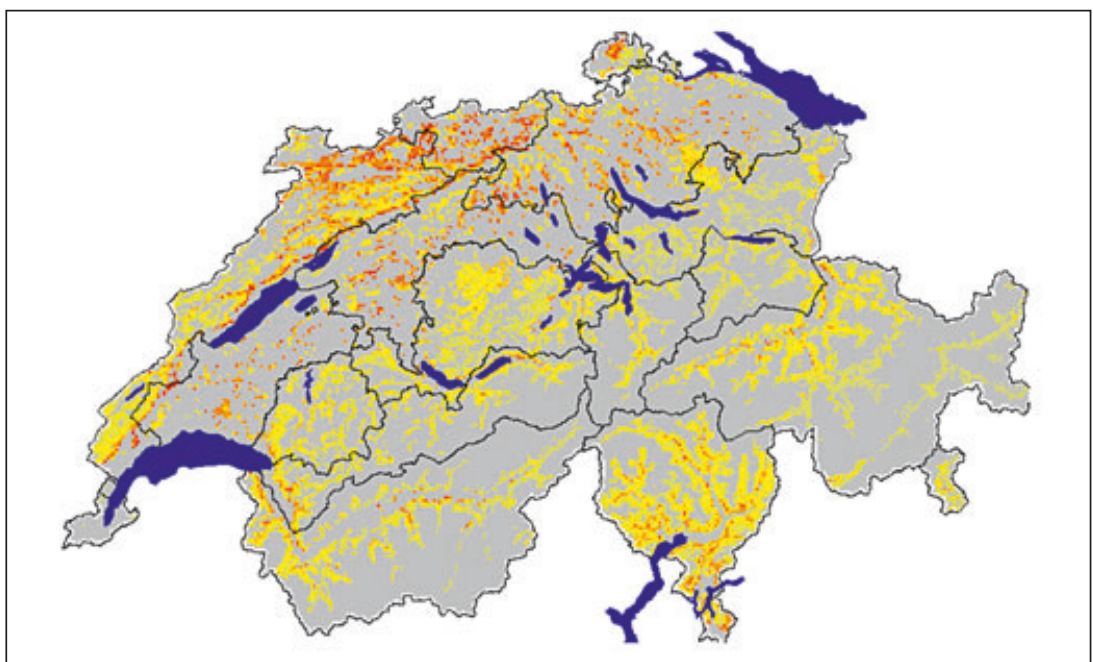

Abb 7 Potenzielle «saproxylische Hotspots» (rot) im Waldareal (gelb). Ergebnis der Kumulation von 55 modellierten Lebensraumeignungskarten für alt- und totholzabhängige Fledermaus-, Vogel-, Amphibien- und Reptilien-, Insekten-, Mollusken-, Pilz- und Flechtenarten (Quelle: Bütler et al 2006).

\section{Diskussion}

Offensichtliche Zusammenhänge zwischen der aktuellen Verbreitung einzelner saproxylischer Arten und dem Vorkommen spezifischer Lebensraumindikatoren gemäss LFI sind eher selten zu beobach- ten. Ein Grund liegt wohl in der beschränkten Repräsentativität der Artkartierungen. Tatsächlich sind systematisch erhobene Artkartierungen die Ausnahme. Bei den meisten Artendatenbanken handelt es sich um die Kumulation von Fundmeldungen, wobei sich intensiveres Suchen in gewissen beliebten Gebieten in einer Häufung von Daten zeigen kann. Ein anderer Grund kann in der geringen Rasterdichte und den kleinen Probeflächen des LFI liegen. Mit der Kumulation von (potenziellen) Arealen mehrerer saproxylischer Arten in Hotspots und der Kumulation von Lebensraumindikatoren im Strukturindex des LFI stehen aber robuste Instrumente für das Lebensraum- und Artenmanagement zur Verfügung. Die Daten des LFI3 belegen, dass alte und zudem seit mindestens 30 Jahren nicht mehr genutzte Wälder eindeutige Hotspots für saproxylische Arten sind. Die Totholzvorräte liegen hier im Bereich der 20 bis $70 \mathrm{~m}^{3}$ pro Hektare, die als Schwellenwerte für das Vorkommen von anspruchsvollen Arten gelten (Müller \& Bütler 2010). Frisch genutzte Bestände weisen deutlich weniger Totholz auf. Am grössten ist das Gefälle diesbezüglich im Areal der Buchenwälder, wo auch die meisten saproxylischen Arten ihr Hauptareal haben. In den letzten zehn Jahren genutzte Bestände auf Buchenwaldstandorten weisen

\begin{tabular}{|c|c|c|c|c|c|c|c|}
\hline \multirow[b]{3}{*}{ Waldgesellschaften } & \multirow[b]{3}{*}{ Holzfestigkeit } & \multicolumn{6}{|c|}{ Totholzvolumen } \\
\hline & & \multicolumn{2}{|c|}{ Alle Bestände } & \multicolumn{2}{|c|}{$\begin{array}{l}\text { Bestände mit Nutzung in den } \\
\text { letzten } 10 \text { Jahren }\end{array}$} & \multicolumn{2}{|c|}{$\begin{array}{l}\text { Bestände älter als } 120 \text { Jahre und } \\
\text { Nutzung vor mehr als } 30 \text { Jahren }\end{array}$} \\
\hline & & $\mathrm{m}^{3} / \mathrm{ha}$ & $\pm \%$ & $\mathrm{~m}^{3} / \mathrm{ha}$ & $\pm \%$ & $\mathrm{~m}^{3} / \mathrm{ha}$ & $\pm \%$ \\
\hline \multirow{2}{*}{ Buchenwälder } & Morsch/Moder/Mull & 4 & 11 & 3 & 16 & 8 & 51 \\
\hline & Total & 16 & 7 & 13 & 9 & 34 & 31 \\
\hline Tannen-Buchen-Wälder & Total & 26 & 8 & 24 & 12 & 39 & 24 \\
\hline \multirow[t]{3}{*}{ Tannen-Fichten-Wälder } & Frisch-/Hartholz & 22 & 11 & 26 & 18 & 31 & 26 \\
\hline & Morsch/Moder/Mull & 10 & 12 & 7 & 23 & 19 & 26 \\
\hline & Total & 32 & 9 & 33 & 16 & 50 & 22 \\
\hline \multirow{2}{*}{$\begin{array}{l}\text { Arven- und } \\
\text { Lärchenwälder }\end{array}$} & Morsch/Moder/Mull & 7 & 18 & 6 & 61 & 13 & 29 \\
\hline & Total & 15 & 14 & 21 & 58 & 25 & 24 \\
\hline \multirow[t]{3}{*}{ Föhrenwälder } & Frisch-/Hartholz & 15 & 18 & 17 & 33 & 19 & 51 \\
\hline & Morsch/Moder/Mull & 9 & 19 & 10 & 39 & 18 & 45 \\
\hline & Total & 24 & 16 & 27 & 29 & 37 & 44 \\
\hline \multirow[t]{3}{*}{ Übrige Laubwälder } & Frisch-/Hartholz & 13 & 8 & 13 & 14 & 14 & 31 \\
\hline & Morsch/Moder/Mull & 5 & 11 & 3 & 20 & 16 & 37 \\
\hline & Total & 18 & 7 & 16 & 13 & 30 & 30 \\
\hline Gesamt & Frisch-/Hartholz & 15 & 4 & 15 & 6 & 22 & 11 \\
\hline
\end{tabular}

Tab 4 Totholzvolumen (Mittelwerte in $\mathrm{m}^{3} / \mathrm{ha}$; Standardfehler in \%) im LFI3 nach Holzfestigkeit, Anzahl Jahren seit dem letzten Eingriff und Bestandesalter. Auswertungseinheit: zugänglicher Wald ohne Gebüschwald 


\begin{tabular}{|c|c|c|c|c|c|c|c|}
\hline \multirow[t]{2}{*}{ Vorrangfunktion } & \multirow[t]{2}{*}{ Inventur } & \multicolumn{2}{|c|}{ Totholzvorrat } & \multicolumn{2}{|c|}{ Anteil Bestände älter 120 Jahre } & \multicolumn{2}{|c|}{$\begin{array}{c}\text { Stammzahlanteil der Habitat } \\
\text { bäume mit Wert }>3\end{array}$} \\
\hline & & $m^{3} / h a$ & $\pm \%$ & $\%$ & $\pm \%$ & $\%$ & $\pm \%$ \\
\hline \multirow[t]{3}{*}{ Holzproduktion } & LFI1 & 2.7 & 9 & 10 & 1 & 19 & 1 \\
\hline & LFI2 & 5.6 & 8 & 19 & 1 & 21 & 1 \\
\hline & LFI3 & 13.1 & 7 & 20 & 1 & 21 & 1 \\
\hline \multirow[t]{3}{*}{ Schutz vor Naturgefahren } & LFI1 & 4.7 & 8 & 10 & 3 & 23 & 1 \\
\hline & LFI2 & 15.1 & 6 & 28 & 1 & 28 & 1 \\
\hline & LFI3 & 25.9 & 6 & 27 & 1 & 27 & 1 \\
\hline \multirow[t]{3}{*}{ Erholung } & LFI1 & 0.9 & 34 & 20 & 1 & 19 & 2 \\
\hline & LFI2 & 2.3 & 33 & 19 & 4 & 25 & 3 \\
\hline & LFI3 & 12.4 & 23 & 17 & 3 & 28 & 3 \\
\hline \multirow[t]{3}{*}{ Naturschutz } & LFI1 & 3.9 & 17 & 14 & 2 & 29 & 2 \\
\hline & LFI2 & 14.8 & 14 & 22 & 2 & 32 & 3 \\
\hline & LFI3 & 21.8 & 14 & 23 & 2 & 32 & 3 \\
\hline \multirow[t]{3}{*}{ Übrige Waldfunktionen } & LFI1 & 2.5 & 28 & 20 & 2 & 20 & 2 \\
\hline & LFI2 & 10.9 & 19 & 29 & 3 & 29 & 3 \\
\hline & LFI3 & 14.5 & 14 & 27 & 3 & 25 & 2 \\
\hline \multirow[t]{3}{*}{ Keine spezielle Waldfunktion } & LFI1 & 3.4 & 29 & 11 & 2 & 20 & 2 \\
\hline & LFI2 & 13.2 & 16 & 22 & 3 & 31 & 3 \\
\hline & LFI3 & 16.5 & 15 & 24 & 3 & 29 & 3 \\
\hline \multirow[t]{3}{*}{ Gesamt } & LFI1 & 3.4 & 5 & 14 & 0 & 21 & 0 \\
\hline & LFI2 & 10.3 & 4 & 23 & 1 & 25 & 0 \\
\hline & LFI3 & 18.5 & 4 & 23 & 1 & 25 & 0 \\
\hline
\end{tabular}

Tab 5 Ökologische Kenngrössen (Mittelwert in $\mathrm{m}^{3} /$ ha respektive \%, Standardfehler in \%) nach Waldfunktionen (Vorrangfunktionen). Infolge unterschiedlicher Definitionen wird der Totholzvorrat im LFI1 um etwa einen Drittel unterschätzt. Übrige Waldfunktionen: landwirtschaftliche Nutzung, Windschutz, Trinkwasserschutz, Landschaftsschutz, Wildschutz, Militär. Auswertungseinheit: gemeinsamer zugänglicher Wald ohne Gebüschwald LFI1/LFI2/LFI3.

durchschnittlich $13 \mathrm{~m}^{3}$ ha Totholz auf, seit mindestens 30 Jahren ungenutzte Altbestände dagegen $34 \mathrm{~m}^{3} / \mathrm{h}$. Dies führt zur Hypothese, dass Nutzungsverzichte schon nach 30 Jahren zu wertvollen Totholzvorräten führen könnten, wie dies Bütler \& Lachat (2009) aufzeigten. Alte, ungenutzte Wälder insbesondere Buchen- und Eichenwälder - sollten folglich prioritär in Naturschutzstrategien berücksichtigt werden, zumal das Alt- und Totholzvorkommen im Gebiet der potenziellen Hotspots von saproxylischen Arten wie gezeigt relativ gering ist.

Die LFI-Daten belegen aber auch, dass Alt- und Totholz innert 20 Jahren ganz erheblich zugenommen haben, und zwar im Naturschutzwald wie auch im Holzproduktionswald und im Wald mit Vorrangfunktion Schutz vor Naturgefahren. Dabei wurde im Holzproduktionswald zwischen LFI2 und LFI3 der gesamte Holzzuwachs genutzt, im Naturschutz- und Schutzwald dagegen war es je weniger als die Hälfte. Dies führt zur Hypothese, dass die Förderung von Alt- und Totholz als Element des integralen Naturschutzes ungeachtet der vorrangigen Waldfunktion seit einiger Zeit bei der Waldbewirtschaftung mit entsprechendem Erfolg praktiziert wird, und dies trotz zunehmender Holznutzung. Daneben haben auch Orkane wie Vivian und Lothar Wirkung gezeigt. Der Totholzvorrat und der Anteil hochwertiger Habitatbäume sind im Naturschutzwald nur gut eineinhalbmal so gross wie im Holzproduktionswald. Auch Wälder mit der Vorrangfunktion Schutz vor Naturgefahren bieten relativ gute Lebensraumqualität. Insbesondere die Totholzvorräte sind hier unter allen Standortbedingungen höher als bei jeder anderen Vorrangfunktion. Die Synergie zwischen Schutzwäldern und der Förderung von Altund Totholzspezialisten sollte erkannt und besser genutzt werden. Gleiches gilt für schlecht erschlossene Wälder sowie solche in steilen und höheren Lagen: Dort finden sich im Durchschnitt die grössten Totholzvorräte - ein gutes Startkapital bei der Anlage von Waldreservaten. Generell tief sind die durchschnittlichen Totholzvorräte trotz dem Orkan Lothar in der kollinen und submontanen Stufe. Hier bestehen Potenziale für Aufwertungsmassnahmen im Holzproduktionswald ebenso wie im Naturschutzwald.

Unsere Auswertungen lassen Hinweise für Biodiversitätsstrategien im Wald zu. Verallgemeinert kann man sagen, dass Erhaltungsmassnahmen (engl. conservation) hauptsächlich in hohen, schlecht erschlossenen Lagen und in alten Wäldern angesetzt werden sollten, Förderungs- oder Aufwertungsmassnahmen (engl. restoration) hingegen in erster Linie in tiefen Lagen, in flachem, gut erschlossenem Gelände mit einem Akzent in Holzproduktionswäldern. Ähnliche Ergebnisse fanden auch Bütler et al (2005). 


\begin{tabular}{|c|c|c|c|c|c|c|c|c|c|c|}
\hline & \multicolumn{8}{|c|}{ Waldfunktion (Vorrangfunktion) } & \multirow{2}{*}{\multicolumn{2}{|c|}{ Gesamtwald }} \\
\hline & \multicolumn{2}{|c|}{ Holzproduktion } & \multicolumn{2}{|c|}{$\begin{array}{c}\text { Schutz vor } \\
\text { Naturgefahren }\end{array}$} & \multicolumn{2}{|c|}{ Naturschutz } & \multicolumn{2}{|c|}{ Übriger Wald } & & \\
\hline & $\mathrm{m}^{3} / \mathrm{ha}$ & $\pm \%$ & $\mathrm{~m}^{3} / \mathrm{ha}$ & $\pm \%$ & $\mathrm{~m}^{3} / \mathrm{ha}$ & $\pm \%$ & $\mathrm{~m}^{3} / \mathrm{ha}$ & $\pm \%$ & $\mathrm{~m}^{3} / \mathrm{ha}$ & $\pm \%$ \\
\hline subalpin & 16.2 & 3.0 & 33.2 & 3.1 & 31.2 & 6.9 & 17.8 & 2.8 & 26.9 & 1.9 \\
\hline montan & 13.8 & 1.3 & 22.9 & 1.8 & 17.3 & 4.1 & 11.6 & 1.7 & 17.0 & 0.9 \\
\hline \multicolumn{11}{|l|}{ Geländeneigung } \\
\hline $1-20 \%$ & 9.8 & 1.0 & 12.1 & 4.1 & 12.4 & 3.2 & 12.4 & 2.8 & 10.4 & 0.9 \\
\hline $21-40 \%$ & 10.9 & 1.3 & 20.1 & 3.9 & 16.6 & 4.9 & 10.0 & 1.8 & 12.9 & 1.1 \\
\hline $41-60 \%$ & 18.2 & 2.9 & 23.2 & 2.5 & 27.5 & 7.4 & 16.2 & 3.1 & 20.6 & 1.6 \\
\hline über $60 \%$ & 24.6 & 4.5 & 29.5 & 2.1 & 29.7 & 7.3 & 19.8 & 3.0 & 27.5 & 1.6 \\
\hline $101-200 \mathrm{~m}$ & 12.3 & 1.6 & 22.3 & 2.9 & 21.5 & 7.4 & 14.1 & 2.9 & 16.7 & 1.4 \\
\hline über $200 \mathrm{~m}$ & 19.5 & 2.7 & 29.4 & 2.1 & 24.4 & 4.6 & 17.3 & 2.1 & 24.5 & 1.3 \\
\hline \multicolumn{11}{|c|}{ Potenzieller Holzernteaufwand } \\
\hline bis $50 \mathrm{CHF} / \mathrm{m}^{3}$ & 11.9 & 1.2 & 25.3 & 6.0 & 17.5 & 5.3 & 10.9 & 2.9 & 13.3 & 1.1 \\
\hline $51-100 \mathrm{CHF} / \mathrm{m}^{3}$ & 14.2 & 1.5 & 26.4 & 2.6 & 21.2 & 4.8 & 13.7 & 2.1 & 18.9 & 1.2 \\
\hline mehr als $100 \mathrm{CHF} / \mathrm{m}^{3}$ & 14.6 & 3.4 & 24.9 & 1.8 & 23.1 & 4.8 & 17.2 & 2.3 & 21.8 & 1.3 \\
\hline \multicolumn{11}{|l|}{ Letzte Nutzung vor } \\
\hline bis zu 10 Jahren & 12.4 & 1.1 & 29.2 & 3.2 & 16.2 & 4.6 & 10.7 & 2.0 & 16.4 & 1.0 \\
\hline 11-20 Jahren & 13.1 & 2.5 & 21.1 & 3.3 & 27.5 & 9.3 & 9.5 & 2.7 & 16.6 & 1.8 \\
\hline mehr als 20 Jahren & 15.4 & 2.1 & 25.5 & 1.8 & 23.1 & 4.1 & 18.3 & 2.1 & 21.7 & 1.1 \\
\hline
\end{tabular}

Tab 6 Totholzvorrat (Mittelwert in $\mathrm{m}^{3} / \mathrm{ha}$, Standardfehler in \%) nach Waldfunktionen und Aspekten des Standortes und der Bewirtschaftung. Übriger Wald: Vorrangfunktionen landwirtschaftliche Nutzung, Windschutz, Trinkwasserschutz, Erholung, Landschaftsschutz, Wildschutz, Militär, keine Vorrangfunktion. Eigentumskategorie: öffentlich mit Steuerhoheit: Bund, Kantone, politische Gemeinden; öffentlich ohne Steuerhoheit: Bürgergemeinden, Korporationen; privat: Gesellschaften, Einzeleigentum. Auswertungseinheit: gemeinsamer zugänglicher Wald ohne Gebüschwald LFI1/LFI2/LFI3.

Angesichts von Szenarien einer verstärkten Holznutzung in der Zukunft ist die Inkraftsetzung von Altund Totholzkonzepten eine wichtige, dringende Massnahme, um eine langfristige gute Lebensraumqualität für Alt- und Totholzspezialisten zu garantieren. Die LFI-Daten sind auch wertvoll im Hinblick auf eine gezieltere Auswahl von potenziellen Waldreservaten, was bisher in Europa noch zu wenig systematisch verfolgt wurde (Branquart et al 2008).

\section{Schlussfolgerung}

Die Erhebungen des LFI sind, insbesondere wenn es sich um gemessene Grössen handelt, gut geeignet, die Veränderung des Lebensraumes Wald in Raum und Zeit zu verfolgen. Auswirkungen von Extremereignissen wie Orkanen, aber auch die unterschiedlichen Standorte und Bewirtschaftungen zei- gen sich in den Lebensraumindikatoren. LFI-Daten bilden eine Grundlage zur Herleitung entsprechender Hypothesen. Direkte Beziehungen zwischen Letzteren und der Verbreitung von Arten(gruppen) lassen sich aus mehreren Gründen kaum nachweisen, dazu sind Fallstudien geeigneter. Hingegen können mit gezielten Aufnahmen gut definierter Merkmale auch künftig weitere, neue Informationen zur Qualität von Habitaten gewonnen werden. Wo die direkte Erfassung solcher Merkmale wie beim Baumalter an praktische Grenzen stösst, können anhand von Messungen auf Unterstichproben Modelle entwickelt werden, wie dies für die häufige Fichte derzeit gemacht wird.

LFI-Daten ermöglichen es, Lebensraum-Hotspots für Alt- und Totholzspezialisten zu identifizieren und Massnahmen für eine effiziente, nationale oder regionale Biodiversitätsförderung zu treffen. Gemäss unseren Beispielen sind Aussagen zu ver- 
schiedenen Bereichen möglich. Erstens können zum Beispiel geografische Schwerpunkte für Erhaltungsoder Förderungsmassnahmen aufgezeigt werden. Zweitens ist es möglich, die zeitliche Entwicklung des Potenzials für saproxylische Arten zu beurteilen (z.B. 30 Jahre ohne Nutzung vs. kürzlich genutzt). Drittens kann das Angebot an Habitatstrukturen, Alt- und Totholz zwischen Waldgesellschaften verglichen werden, um daraus Prioritäten für Schutzprogramme abzuleiten (z.B. Buchenwälder als Förderungsschwerpunkt). Viertens können Synergien beziehungsweise Konflikte zwischen Waldvorrangfunktionen und der Lebensraumqualität für Alt- und Totholzspezialisten aufgezeigt werden (z.B. die Synergie mit dem Schutz vor Naturgefahren oder der Konflikt mit der Holzproduktionsfunktion). Als Fazit ergibt sich, dass die LFI-Daten vermehrt für solche Zwecke genutzt werden sollten.

Eingereicht: 31. Oktober 2010, akzeptiert (mit Review): 23. Mai 2011

\section{Literatur}

BÖHL J, BRÄNDLI UB (2007) Deadwood volume assessment in the third Swiss National Forest Inventory: methods and first results. Eur J For Res 126: 449-457.

BOLLMANN K ET AL (2009) Konzepte, Instrumente und Herausforderungen bei der Förderung der Biodiversität im Wald. Schweiz Z Forstwes 160: 53-67. doi: 10.3188/ szf. 2009.0053

BRÄNDLI UB, EDITOR (2010) Schweizerisches Landesforstinventar. Ergebnisse der dritten Erhebung 2004-2006. Birmensdorf: Eidgenöss Forsch.anstalt Wald Schnee Landschaft. $312 \mathrm{p}$.

BRÄNDLI UB, ABEGG M, DUC P, GINZLER C (2010) Biologische Vielfalt. In: Brändli UB, editor. Schweizerisches Landesforstinventar. Ergebnisse der dritten Erhebung 20042006. Birmensdorf: Eidgenöss Forsch.anstalt Wald Schnee Landschaft. pp. 187-228.

BRÄNDLI UB, ABEGG M, BERANOVA J (2011) Forest age. In: Chirici $G$, Winter S, McRoberts RE, editors. National forest inventories: Contributions to forest biodiversity assessments. Berlin: Springer. pp. 145-160.

BRÄNDLI UB, BÜHLER C, ZANGGER A (2007A) Biodiversität und Waldinventuren. Birmensdorf: Eidgenöss Forsch.anstalt Wald Schnee Landschaft, LFI Info 7. pp. 1-6.

BRÄNDLI UB, BÜHLER C, ZANGGER A (2007B) Waldindikatoren zur Artenvielfalt - Erkenntnisse aus LFI und BDM Schweiz. Schweiz Z Forstwes 158: 243-254. doi: 10.3188/ szf. 2007.0243

BRÄNDLI UB, DOWHANYTSCH J, EDITORS (2003) Urwälder im Zentrum Europas. Ein Naturführer durch das KarpatenBiosphärenreservat in der Ukraine. Bern: Haupt. 192 p.

BRANQUART E, VERHEYEN K, LATHAM J (2008) Selection criteria of protected forest areas in Europe: The theory and the real world. Biol Conserv 141: 2795-2806.

BUSSLER H (2010) Hotspot-Gebiete xylobionter Urwaldreliktarten aus dem Reich der Käfer. Freising: Bayerische Landesanstalt Wald Forstwirtschaft, LWF aktuell 76. pp. 10-12.

BÜTLER R, LACHAT T, SCHLAEPFER R (2005) Grundlagen für eine Alt- und Totholzstrategie der Schweiz. Lausanne: EPF Lausanne, Laboratorium Ökosystemmanagement. 100 p.
BÜTLER R, LACHAT T, SCHLAEPFER R (2006) Saproxylische Arten in der Schweiz: ökologisches Potential und Hotspots. Schweiz Z Forstwes 157: 208-216. doi: 10.3188/ SZf.2006.0208

BÜTLER R, LACHAT T (2009) Wälder ohne Bewirtschaftung: eine Chance für die saproxylische Biodiversität. Schweiz Z Forstwes 160: 324-333. doi: 10.3188/szf.2009.0324

BUWAL, WSL (2005) Waldbericht 2005 - Zahlen und Fakten zum Zustand des Schweizer Waldes. Bern: Bundesamt Umwelt Wald Landschaft. 152 p.

GJERDE I, SAETERSDAL M, BLOM HH (2007) Complementary hotspot inventory - a method for identification of important areas for biodiversity at the forest stand level. Biol Conserv 137: 549-557.

новонм С (2005) Was sind Biodiversity Hotspots - global, regional, lokal? Tuexenia 25: 379-386.

KELLER M, EDITOR (2010) Schweizerisches Landesforstinventar. Feldaufnahme-Anleitung 2010. Birmensdorf: Eidgenöss Forsch.anstalt Wald Schnee Landschaft. 213 p.

KOORDINATIONSSTELLE BIODIVERSITÄTS-MONITORING SCHWEIZ (2009) Zustand der Biodiversität in der Schweiz. Ergebnisse des Biodiversitäts-Monitorings Schweiz (BDM) im Überblick. Stand: Mai 2009. Bern: Bundesamt Umwelt, Umwelt-Zustand 0911. 112 p.

LACHAT T, BÜTLER R (2007) Gestion des vieux arbres et du bois mort - îlots de sénescence, arbres-habitat et métapopulations saproxyliques. Lausanne: EPF Lausanne, Laboratoire des systèmes écologiques, Forschungsbericht. 81 p. MEIER S (2009) Beurteilung des ökologischen und ökonomischen Wertes von Habitatbäumen in Wirtschaftswäldern am Beispiel des Forstbetriebes Baden. Zürich: ETH Zürich, Departement Umweltwissenschaften, Masterarbeit. $107 \mathrm{p}$.

MEYER P, SCHMIDT M, SPELLMANN H (2009) Die «HotspotsStrategie» - Wald-Naturschutzkonzept auf landschaftsökologischer Grundlage. Allg Forst Z Waldwirtsch Umweltvorsorge 15: 822-824.

MONING C, MÜLLER J (2009) Critical forest age thresholds for the diversity of lichens, molluscs and birds in beech (Fagus sylvatica L.) dominated forests. Ecol Indic 9: 922-933.

MÜLLER J (2005) Waldstrukturen als Steuergrösse für Artengemeinschaften in kollinen bis submontanen Buchenwäldern. Weihenstephan: Techn Univ München, Department Ökosystem- Landschaftsmanagement, Dissertation. 228 p.

MÜLLER J ET AL (2005) Urwald relict species - saproxylic beetles indicating structural qualities and habitat tradition. www.afsv.de/download/literatur/waldoekologie-online/ waldoekologie-online_heft-2-9.pdf (25.5.2011)

MÜLLER J, BÜTLER R (2010) A review of habitat thresholds for dead wood: a baseline for management recommendations in European forests. Eur J Forest Res 129: 981-992.

MYERS N, MITTERMEIER RA, MITTERMEIER CG, DA FONSECA GAB, KENT J (2000) Biodiversity hotspots for conservation priorities. Nature 403: 853-858.

NIEDERMANN-MEIER S, MORDINI M, BÜTLER R, ROTACH P (2010) Habitatbäume im Wirtschaftswald: ökologisches Potenzial und finanzielle Folgen für den Betrieb. Schweiz Z Forstwes 161: 391-400. doi: 10.3188/szf.2010.391

PROJEKTLEITUNG WAP-CH, BHP BRUGGER (2004) Waldprogramm Schweiz (WAP-CH). Bern: Bundesamt Umwelt Wald Landschaft, Schriftenreihe Umwelt 363. 117 p.

SBN (1989) Thesen für mehr Natur im Wald. Basel: Schweiz Bund Naturschutz, Beitr Nat.schutz Schweiz 11. 74 p.

SCHMID H, LUDER R, NAEF-DAENZER B, GRAF R, ZBINDEN N (1998) Schweizer Brutvogelatlas. Verbreitung der Brutvögel in der Schweiz und im Fürstentum Liechtenstein 1993-1996. Sempach: Schweizerische Vogelwarte. 574 p. 
STOFER S ET AL (2011) SwissLichens - Webatlas der Flechten der Schweiz / Modul Verbreitung (Version 2). www.swisslichens.ch (22.5.2011)

WINTER S, SCHUMACHER H, FLADE M, MÖLLER G (2003) Naturschutzstandards für die Bewirtschaftung von Buchenwäldern im nordostdeutschen Tiefland. Sachbericht der Landesanstalt für Grossschutzgebiete über das F \& E-Vorhaben
«Biologische Vielfalt und Forstwirtschaft». Brandenburg: Landesanstalt Grossschutzgebiete, Forschungsbericht. $445 \mathrm{p}$.

WINTER S, MÖLLER GC (2008) Microhabitats in lowland beech forests as monitoring tool for nature conservation. For Ecol Manage 255: 1251-1261.

\section{Lebensraum-Hotspots für saproxylische Arten mittels LFI-Daten erkennen}

Alt- und Totholz sind die wichtigsten Lebensraumaspekte für Tausende typischer waldbewohnender Tier-, Pilz- und Pflanzenarten. Dieser Beitrag geht der Frage nach, ob mit Daten des Landesforstinventars (LFI) sogenannte Lebensraum-Hotspots für eine effiziente nationale und regionale Artenförderung ermittelt werden können. Dazu wurden die für Alt- und Totholzspezialisten wichtigsten Lebensraumindikatoren Altholz, Totholz und Habitatbäume anhand von LFI-Daten analysiert sowie aus allen drei Indikatoren ein Strukturindex berechnet. Die Ergebnisse zeigen, dass sich nationale Verbreitungskarten ausgewählter saproxylischer Arten in unterschiedlichem Mass mit dem Vorkommen von Lebensraumindikatoren gemäss LFI decken, oftmals ein Effekt der nicht repräsentativen Fundmeldungen. Deshalb wurden die Indikatoren auch mit modellierten Verbreitungsgebieten verglichen, den potenziellen Hotspots der saproxylischen Arten. Dabei wird ersichtlich, dass besonders das Totholz heute selten dort häufig ist, wo es zur Erhaltung der Biodiversität am meisten von Nutzen wäre. Besonders hoch sind die Totholzvorräte in den westlichen Randalpen. Schwerpunkte der Altbestände liegen im Engadin und im Wallis, grosse Hotspots der Habitatbäume im Tessin, im zentralen Jura und im Unterwallis.

Mit LFI-Daten zu Standort, Bewirtschaftung und vorrangiger Waldfunktion wird der Einfluss dieser Faktoren gezeigt. So weisen Tannen-Fichten-Wälder heute doppelt so viel Totholz auf wie Buchenwälder ( 32 vs. $16 \mathrm{~m}^{3} / \mathrm{ha}$ ), erstere in seit über 30 Jahren ungenutzten Altbeständen $50 \mathrm{~m}^{3} / \mathrm{ha}$. Unabhängig von der Waldfunktion sind die Totholzvorräte in höheren Lagen, in steilerem Gelände und in schlechter erschlossenen Wäldern grösser. Schutzwälder weisen am meisten Altbestände (27\% älter als 120 Jahre) und Totholz auf ( $\left.26 \mathrm{~m}^{3} / \mathrm{ha}\right)$, während Erholungs- und Holzproduktionswälder 12 bis $13 \mathrm{~m}^{3} /$ ha Totholz und 17 bis $20 \%$ Altbestände haben. In den letzten zwei Jahrzehnten haben Alt- und Totholz bei allen Waldfunktionen erheblich zugenommen.

Die Erhebungen des LFI sind gut geeignet, um die Veränderung des Lebensraumes Wald in Raum und Zeit zu verfolgen sowie die Einflüsse von Standort, Bewirtschaftung und Naturereignissen aufzuzeigen. Differenzierte LFI-Datenanalysen bieten Hinweise für Naturschutzstrategien, zum Beispiel geografische Schwerpunkte für Erhaltungs- oder Förderungsmassnahmen, das Potenzial von seit längerer Zeit ungenutzten Wäldern oder die Synergie mit Schutzwäldern. LFI-Daten sollten vermehrt für solche Zwecke genutzt werden.

\section{Identifier les hotspots d'habitat pour les espèces saproxyliques à l'aide des données de I'IFN}

Le bois mort et les vieux arbres figurent parmi les composantes d'habitat primordiales pour des milliers d'espèces d'animaux, de champignons et de plantes forestières. Cet article poursuit l'objectif de vérifier si la mise en évidence des hotspots d'habitat est possible à l'aide des données de I'Inventaire forestier national (IFN). Nous avons analysé les indicateurs vieux arbres, bois mort et arbres-habitat, et calculé un nouvel indicateur structurel issu des trois derniers. Les résultats montrent que les cartes de distribution des espèces saproxyliques choisies, basées sur des observations parfois non représentatives, ne coïncident que partiellement avec les cartes des indicateurs mentionnés issues de l'IFN. C'est pourquoi nous avons également comparé les indicateurs aux hotspots potentiels modélisés d'espèces saproxyliques. On peut constater que le bois mort est souvent rare aux endroits ayant le plus grand potentiel pour la biodiversité. Les volumes de bois mort les plus élevés se trouvent en bordure des Alpes de I'Ouest. Les vieux peuplements ont leur centre de distribution en Engadine et en Valais. Les hotspots des arbres-habitat sont localisés au Tessin, au Jura central et en Bas-Valais. Nous avons également analysé l'influence de la station, de la gestion et de la fonction forestière prépondérante sur les indicateurs. Le volume de bois mort des sapinières-pessières est deux fois plus grand que celui des hêtraies ( $32 \mathrm{vs} 16 \mathrm{~m}^{3} / \mathrm{ha}$ ). Les sapinières-pessières sans exploitation depuis au moins 30 ans contiennent $50 \mathrm{~m}^{3} / \mathrm{ha}$. Quelque soit la fonction prépondérante, les volumes de bois mort sont plus grands en altitude, dans les forêts pentues ou mal desservies. Les forêts protectrices sont les plus riches en vieux arbres (27\% plus vieux que 120 ans) et en bois mort ( $\left.26 \mathrm{~m}^{3} / \mathrm{ha}\right)$, tandis que les forêts d'accueil et de production contiennent $17-20 \%$ de vieux peuplements et $12-13 \mathrm{~m}^{3} /$ ha de bois mort. Durant les dernières deux décennies, les quantités de bois mort et de vieux arbres ont considérablement augmenté.

Les données de I'IFN se prêtent bien au suivi des modifications spatio-temporelles de l'habitat forestier, ainsi qu'à la mise en évidence de l'influence de la station et de la gestion forestière. Des analyses différenciées de ces données peuvent fournir des indications utiles pour les stratégies de protection de la nature, tels que par exemple les centres de gravité géographiques pour des mesures de conservation et de restauration, le potentiel des forêts non exploitées depuis longtemps, ou les synergies avec les forêts protectrices. Les données de I'IFN devraient être exploitées davantage à de telles fins. 